\title{
Exosomes derived from Fusobacterium nucleatum- infected colorectal cancer cells facilitate tumour metastasis by selectively carrying miR-1246/92b- 3p/27a-3p and CXCL16
}

\author{
Songhe Guo, ${ }^{1}$ Jun Chen, ${ }^{1}$ Fangfang Chen, ${ }^{1}$ Qiuyao Zeng, ${ }^{2,3}$ Wan-Li Liu, ${ }^{2,3}$ \\ Ge Zhang (D) ${ }^{1}$
}

'Department of Microbial and Biochemical Pharmacy, School of Pharmaceutical Sciences, Sun Yat-sen University, Guangzhou, China

${ }^{2}$ Department of Clinical Laboratory Medicine, Sun Yatsen University Cancer Center, Guangzhou, Guangdong, China ${ }^{3}$ State Key Laboratory of Oncology in South China, Collaborative Innovation Center for Cancer Medicine, Sun Yatsen University Cancer Center, Guangzhou, Guangdong, China

\section{Correspondence to}

Dr Ge Zhang, Department of Microbial and Biochemical Pharmacy, Sun Yat-sen University, Guangzhou, China; zhangge@mail.sysu.edu.cn and Dr Wan-Li Liu, Department of Clinical Laboratory Medicine Department of Clinical Laboratory Medicine, Sun Yatsen University cancer center, Guangzhou, Guangdong, China; liuwl@sysucc.org.cn

GZ and W-LL contributed equally.

Received 22 March 2020 Revised 20 October 2020 Accepted 25 October 2020 Published Online First 10 November 2020

\section{Linked}

- http://dx.doi.org/10.1136/ gutjnl-2020-323496

Check for updates

(C) Author(s) (or their employer(s)) 2021. No commercial re-use. See rights and permissions. Published by BMJ.

To cite: Guo S, Chen J,

Chen F, et al. Gut

2021;70:1507-1519.

\section{ABSTRACT}

Objective Exosomes released from tumour cells are packed with unique RNA and protein cargo, and they are emerging as an important mediator in the communication network that promotes tumour progression. The facultative intracellular bacterium Fusobacterium nucleatum (Fn) is an important colorectal cancer (CRC)-associated bacterium. To date, the function of exosomes from Fn-infected CRC cells has not been explored.

Design Exosomes were isolated by sequential differential centrifugation and verified by transmission electron microscopy, NanoSight analysis and Western blotting. Given that exosomes have been shown to transport miRNAs and proteins to alter cellular functions, we performed miRNA sequencing and proteome analysis of exosomes from Fn-infected and non-infected cells. The biological role and mechanism of exosomes from Fninfected cells in CRC tumour growth and liver metastasis were determined in vitro and in vivo.

Results We demonstrated that exosomes delivered miR-1246/92b-3p/27a-3p and CXCL16/RhoA/IL-8 from Fn-infected cells into non-infected cells to increase cell migration ability in vitro and promote tumour metastasis in vivo. Finally, both circulating exosomal miR-1246/92b$3 p / 27 a-3 p$ and CXCL16 levels were closely associated with Fn abundance and tumour stage in patients with CRC

Conclusion This study suggests that Fn infection may stimulate tumour cells to generate miR-1246/92b3p/27a-3p-rich and CXCL16/RhoA/IL-8 exosomes that are delivered to uninfected cells to promote prometastatic behaviours.

\section{INTRODUCTION}

Exosomes are extracellular vesicles (EVs) produced by various cell types via endocytosis and released into the extracellular milieu. ${ }^{1}$ Exosomes contain a wide range of functional lipids, proteins, RNA and DNA that can be transferred horizontally to recipient cells. ${ }^{2}$ Tumour-derived exosomes (TEXs) carry cargo reflecting the genetic or signalling alterations in the cancer cells of origin and they serve in the communication network of the tumour. ${ }^{3}$ TEXs have been recently implicated in the tumour metastatic process via the transfer of miRNA and proteins
Significance of this study

What is already known on this subject?

- Fusobacterium nucleatum (Fn) is the most important microbe-related risk factor in the initiation and progression of colorectal cancer (CRC).

- Fn is a facultative intracellular bacterium.

- Tumour-derived exosomes (TEXs) carry cargo reflecting genetic or signalling alterations in the cancer cells of origin and serve in the communication network of tumours.

What are the new findings?

- Fn infection increases the secretion of exosomes from CRC cells.

- Fn infection stimulates tumour cells to generate miR-1246/92b-3p/27a-3p-enriched and CXCL16/RhoA/IL-8-enriched exosomes.

- Exosomes secreted from Fn-infected cells promote CRC metastasis.

- Patients with CRC had significantly higher levels of circulating exosomal miR-1246/92b$3 p / 27 a-3 p$ and CXCL16 than healthy subjects.

How might it impact on clinical practice in the foreseeable future?

- As exosomes from Fn-infected CRC cells function as an oncogenic factor promoting CRC development and metastasis, it may be a promising therapeutic strategy to eradicate $\mathrm{Fn}$ infection to treat CRC.

to normal tissues. ${ }^{5}$ Specific proteins present in TEXs have been reported to be promoters of metastatic pathways and to determine organ-specific metastasis. ${ }^{6}$ TEXs were also shown to target nontransformed cells in premetastatic organs and to modulate premetastatic organ cells predominantly through transferred miRNAs. ${ }^{7}$ These dysregulated miRNAs and proteins, which can be delivered to local and distal cells by exosomes play a critical role in tumour metastasis.

Chronic inflammation and infection are the most important epigenetic and environmental factors contributing to tumourigenesis and tumour 
progression. During viral, bacterial, parasitic and fungal infections, pathogens secrete exosomes to spread infection and avoid the host immune system and also stimulate exosomes production in host cells to regulate host immune responses. ${ }^{8}$ Host-derived vesicles carry specific RNA and protein from both pathogens and hosts. Several studies have reported that Mycobacterium-miRNA can repattern host metabolism machinery to favour its intracellular survival. ${ }^{9}$ Exosomes derived from CagA-positive H. pylori infection were reported to promote macrophage-derived foam cell formation. ${ }^{10}$

Fusobacterium nucleatum (Fn) is an obligate anaerobic oncogenic bacterium that is the most important microbe-related risk factor in the initiation and progression of colorectal cancer (CRC). ${ }^{11} 12$ The elucidated mechanisms of Fn involved in colorectal carcinogenesis include immune modulation (increasing myeloid-derived suppressor cells and inhibitory receptors of natural killer cells), virulence factors (FadA and Fap2), microRNAs (miR-21) and bacterial metabolism. ${ }^{13-15}$ Our previous study indicated that $\mathrm{Fn}$ is a facultative intracellular bacterium that can survive in macrophages. ${ }^{16}$ However, whether specific exosomes can be derived from Fn-infected cells and what affects exosome-mediated communication between Fn-infected cells and recipient cells have not been investigated.

In the present study, we investigated the exosomal microRNA and protein profiles during Fn infection and described the role of exosomes secreted from Fn-infected cells on CRC progression and development.

\section{MATERIALS AND METHODS}

\section{Patients and specimen collection}

The serum and stool samples from 82 patients with primary CRC were selected from an archive of blood samples at the Cancer Center of Sun Yat-sen University (SYSUCC) as previously described. ${ }^{17} 18$ In addition, healthy blood samples were obtained from 102 subjects without any malignancy. Background grouping of the study cohorts is shown in online supplemental figure S1.

\section{Exosome isolation, characterisation and nanoparticle tracking analysis}

Exosomes were isolated by differential centrifugation. The exosome size and number were measured by a nanoparticle tracking analysis (NTA) using a Zetasizer Nano S90 system (Malvern Instruments, England) equipped with a blue laser $(405 \mathrm{~nm})$. Purified exosomes were observed with a JEM1400 transmission electron microscope (TEM) operated at $120 \mathrm{kV}$ (JEOL, Japan).

\section{RNA isolation and quantitative RT-PCR}

Total RNA from exosomes was extracted using a total exosome RNA and protein isolation kit (Thermo Fisher Scientific, USA) according to the instructions of the manufacturer. Quantification of miRNA expression was performed using a Mir-X miRNA RT-qPCR TB Green Kit (TaKaRa, Japan) on a Light Cycler 480 II (Roche, Applied Science). The primers were used as shown in online supplemental table S1.

\section{Cell transfection with miRNA mimics or inhibitors}

Cells were transfected with miR-1246/27a-3p/92a-3p mimics, inhibitors or negative control (NC) chemical synthesis oligonucleotides (Gene Pharma, China) using Lipofectamine 3000 reagent (Invitrogen, USA) at the indicated concentrations according to the supplier's instructions. The sequences were used as shown in online supplemental table S2.
Mass spectrometry analysis of exosome proteins

Total protein from exosomes was digested by Pierce Trypsin/ Lys-C Protease Mix, MS-Grade (Thermo Fisher Scientific, USA) and then protein digestion was conducted by using NanoLC-MS/MS analyses (Thermo Fisher Scientific, USA).

\section{Experimental mouse techniques}

To establish a human CRC xenograft model, 6-week-old BALB/c nude mice were subcutaneously inoculated with $2 \times 10^{6}$ HCT116 cells. To establish a mouse CRC homograft model, 6-week-old $\mathrm{BALB} / \mathrm{c}$ mice were inoculated with $2 \times 10^{6} \mathrm{CT} 26$ cells by tail vein injection. After tumour transplantation, phosphate buffer saline (PBS), si-Ex, Ex or Fn-Ex was injected intratumourally or intravenously every other day. The mice were sacrificed 2 weeks after the treatment began, and the tumours were surgically removed and counted. All of the animal studies were conducted in accordance with protocols approved by the Institutional Animal Care and Research Advisory Committee.

\section{Statistical analyses}

Statistical analyses were performed using GraphPad 6.0. Significance was considered $\mathrm{p}<0.05$.

The detailed methodology can be found in online supplemental information.

\section{RESULTS}

\section{Fn infection increases the secretion of exosomes from CRC} cells

To study the specific Fn-infection associated exosomes from CRC cells, HCT116 cells were infected with live Fn at an MOI of 10:1 (bacteria:cells) and then were cultured for 48 hours. Then, exosomes from the supernatant of Fn-infected HCT116 cells (Fn-Ex) were isolated, and exosomes from uninfected HCT116 cells (Ex) and exosomes from E. coli-infected HCT116 cells (Ec-Ex) were used as controls. TEM analysis showed that these purified vesicles samples were oval and globular (figure 1A, online supplemental figure S2A). The NTA revealed that Fn-Ex has a slightly bigger size and higher number than Ex (average $117.4 \pm 35.5$ vs $90.2 \pm 40.6 \mathrm{~nm} ; 6.18 \pm 1.69$ vs $4.59 \pm 1.5210^{8}$ particles $/ 10^{7}$ cells, both $\mathrm{p}<0.05$ ) (figure $1 \mathrm{~B}$ ), while Ex and Ec-Ex are similar in size and number (online supplemental figure S2B). Furthermore, the presence of exosome biomarkers (CD9 and CD63) were verified by Western blotting in Fn-Ex, Ex and Ec-Ex (figure 1C, online supplemental figure S2C). Fn infection markedly increased the secretion of exosomes which were collected from the same volume culture supernatant by Western blotting analysis (figure 1D). Then, $1 \times 10^{5}$ HCT116 cells were incubated with $10 \mu \mathrm{g}$ of PKH26-stained Fn-Ex for 0, 6 and 12 hours. Confocal imaging showed that Fn-Ex was indeed taken up by HCT16 cells at 6-hour postincubation (figure 1E,F).

These data indicate that Fn infection is able to increase the secretion of exosomes from CRC cells and that the infected cells can secrete slightly larger exosomes.

\section{Exosomes derived from Fn-infected cells promote the migration of CRC cells}

To determine the effect of exosomes released by Fn-infected cells on recipient cells, we incubated Fn-Ex, Ex and Ec-Ex with HCT116 and SW480 cells, respectively. CCK8 assays showed that HCT116 and SW480 cells exhibited a significant toxicity following Ec-Ex treatment $(\mathrm{p}<0.05)$, while a slight but not significant toxicity following Fn-Ex or Ex treatment (figure 2A). Interestingly, both Fn-Ex treated HCT116 and SW480 cells 
A

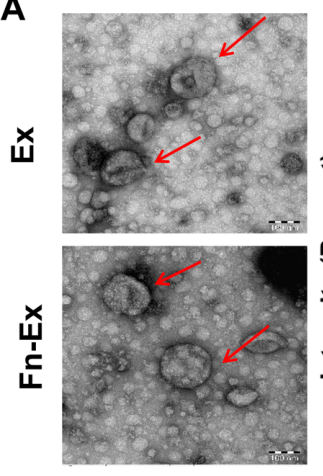

C
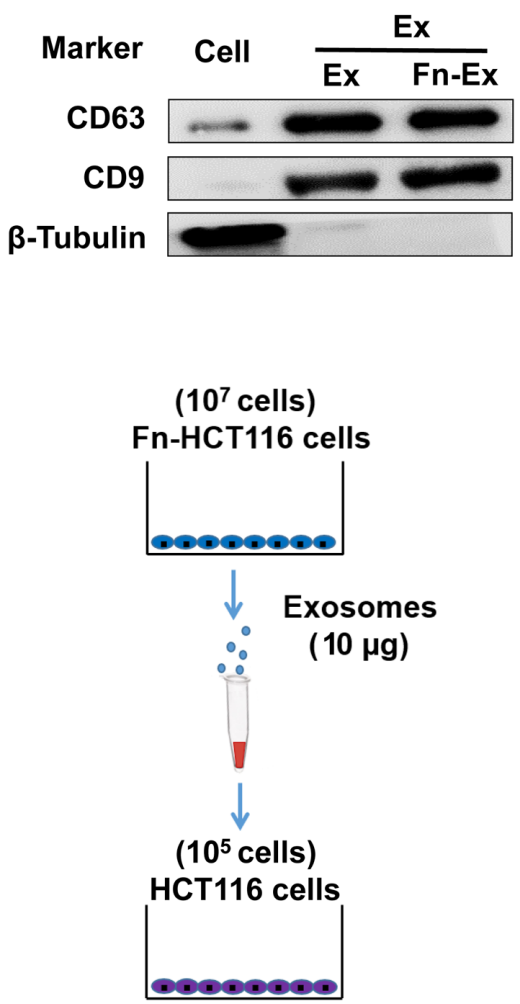

\section{B}
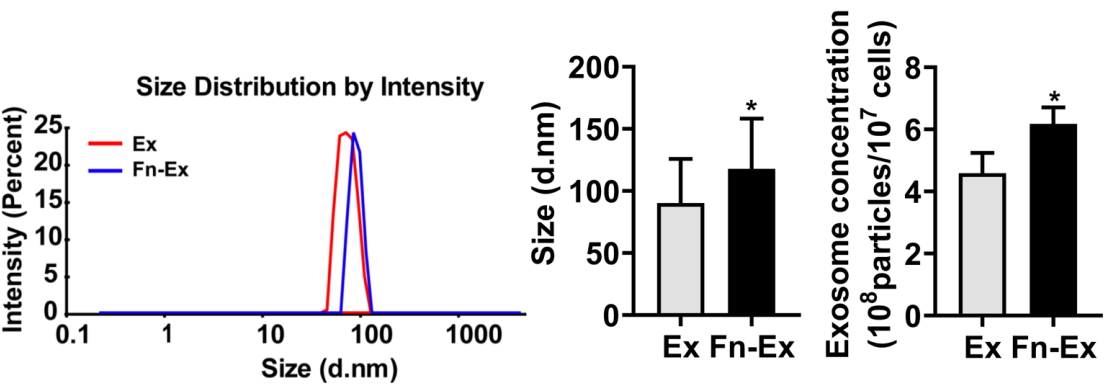

D
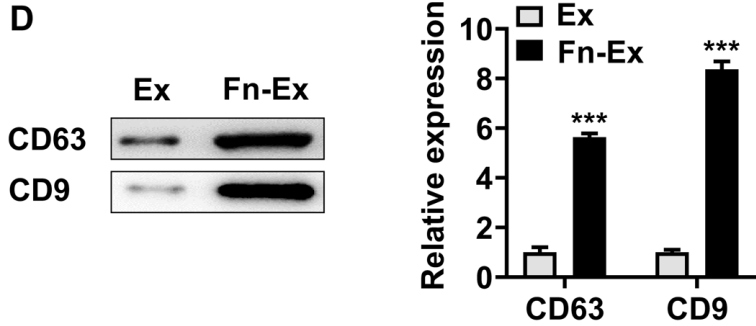

$\mathbf{F}$

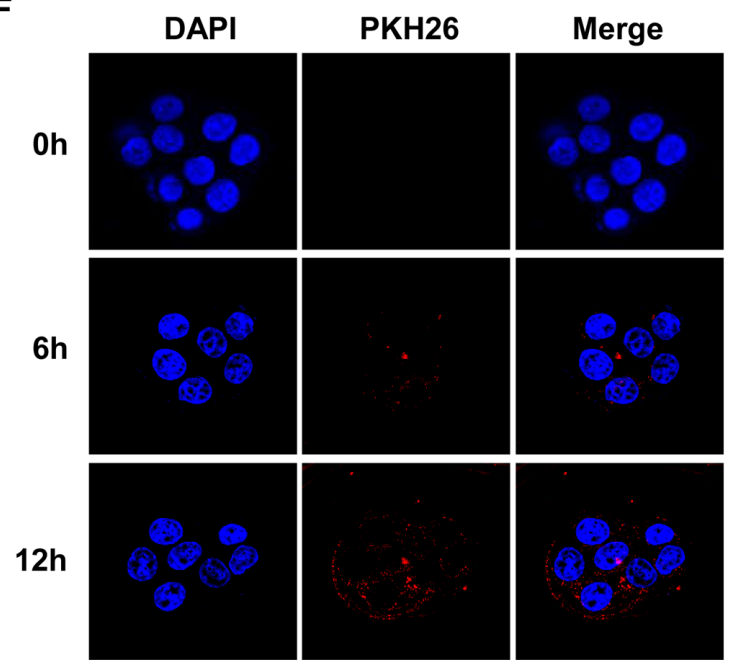

Figure 1 Identification of exosomes derived from HCT116 cells (Ex) and Fn-infected HCT116 cells (Fn-Ex). (A) TEM images of purified Ex and Fn-Ex. Scale bar $=100 \mathrm{~nm}$. (B) NTA of number and size distribution in Ex and Fn-Ex (n=3). (C) Western blot analysis of markers (CD63 and CD9) in exosomal protein purified from HCT116 cells and Fn-infected HCT116 cells supernatants, tubulin served as an internal control in whole-cell lysates. (D) Western blot analysis (left) of Ex and Fn-Ex proteins (CD63 and CD9) collected from the same volume HCT116 cells culture supernatant and quantitative analysis (right). (E) Schematic description of the experimental design. Fn-Ex were isolated and then $1 \times 10^{5}$ HCT116 cells were incubated with $10 \mu \mathrm{g}$ of PKH26-stained Fn-Ex for the indicated time. (F) Confocal microscopy image of the internalisation of fluorescently labelled exosomes in HCT116 cells for the indicated times. Scale bars $=50 \mathrm{~mm}$; Error bars, SD: ${ }^{*} p<0.05 ;{ }^{* *} \mathrm{*}<0.001$. CRC, colorectal cancer; NTA, nanoparticle tracking analysis.

exhibited obvious morphological changes into spindle shapes, which was different from that of the Ex-treated or Ec-Ex-treated cells (figure 2B).

Furthermore, Transwell migration assays showed that Fn-Ex treatment significantly promoted HCT116 cells migration to levels that were not observed in the Ex and Ec-Ex treatment ( 2.9 fold, $\mathrm{p}<0.001 ; 3.9$ fold, $\mathrm{p}<0.001)$. A similar trend was also observed when SW480 cells were recipient cells ( 2.3 fold, $\mathrm{p}<0.001 ; 3.4$ fold, $\mathrm{p}<0.001$ ) (figure $2 \mathrm{C}$ ). Additionally, scratch wounding assays further showed that Fn-Ex treatment significantly accelerated wound closure in both HCT116 and SW480 cells compared with Ex and Ec-Ex treatment (average 65.8\% vs $10.3 \%$ and $10.1 \%, \mathrm{p}<0.001 ; 76.9 \%$ vs $16.3 \%$ and $6.7 \%$, $\mathrm{p}<0.001$, respectively) (figure 2D).
These results showed that exosomes derived from Fn-infected cells change the cell morphology and promote the migration of CRC cells, suggesting that recipient cells may be remodelled by exosomes from Fn-infected cells.

Specific miRNAs are enriched in exosomes secreted by $\mathrm{Fn}$ infected CRC cells

Next, the differential microRNA expression profile of Fn-Ex and Ex was investigated by miRNA-seq analysis. Online supplementary table S3 shows the reads identified for the small RNA and unannotated RNA categories. The percentages of miRNAs in the total RNA isolated from Fn-Ex and Ex were $16.55 \%$ and $13.26 \%$, respectively (figure 3A). A total of 277 and 211 types 
A

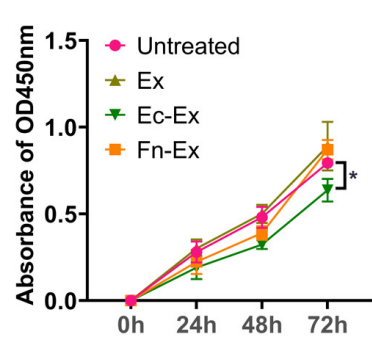

HCT116

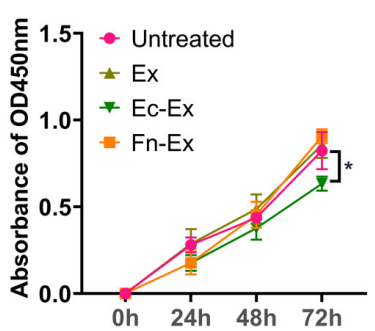

SW480

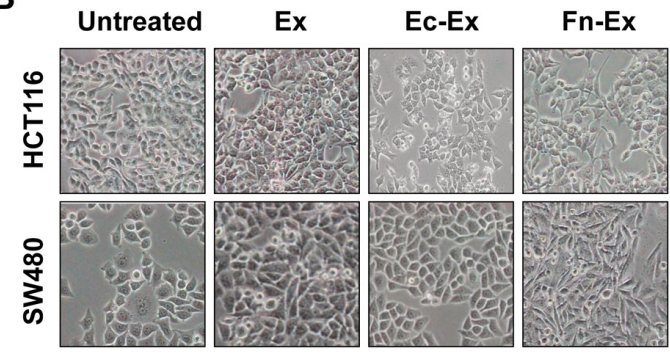

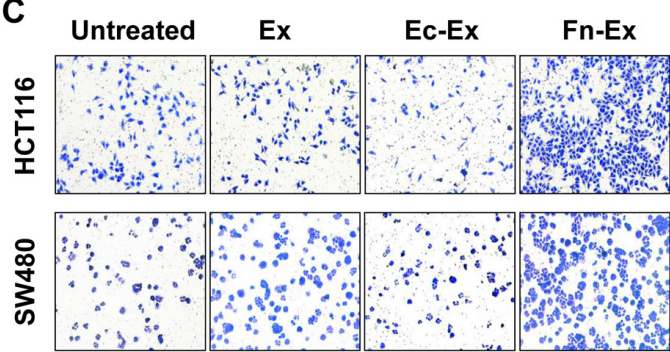
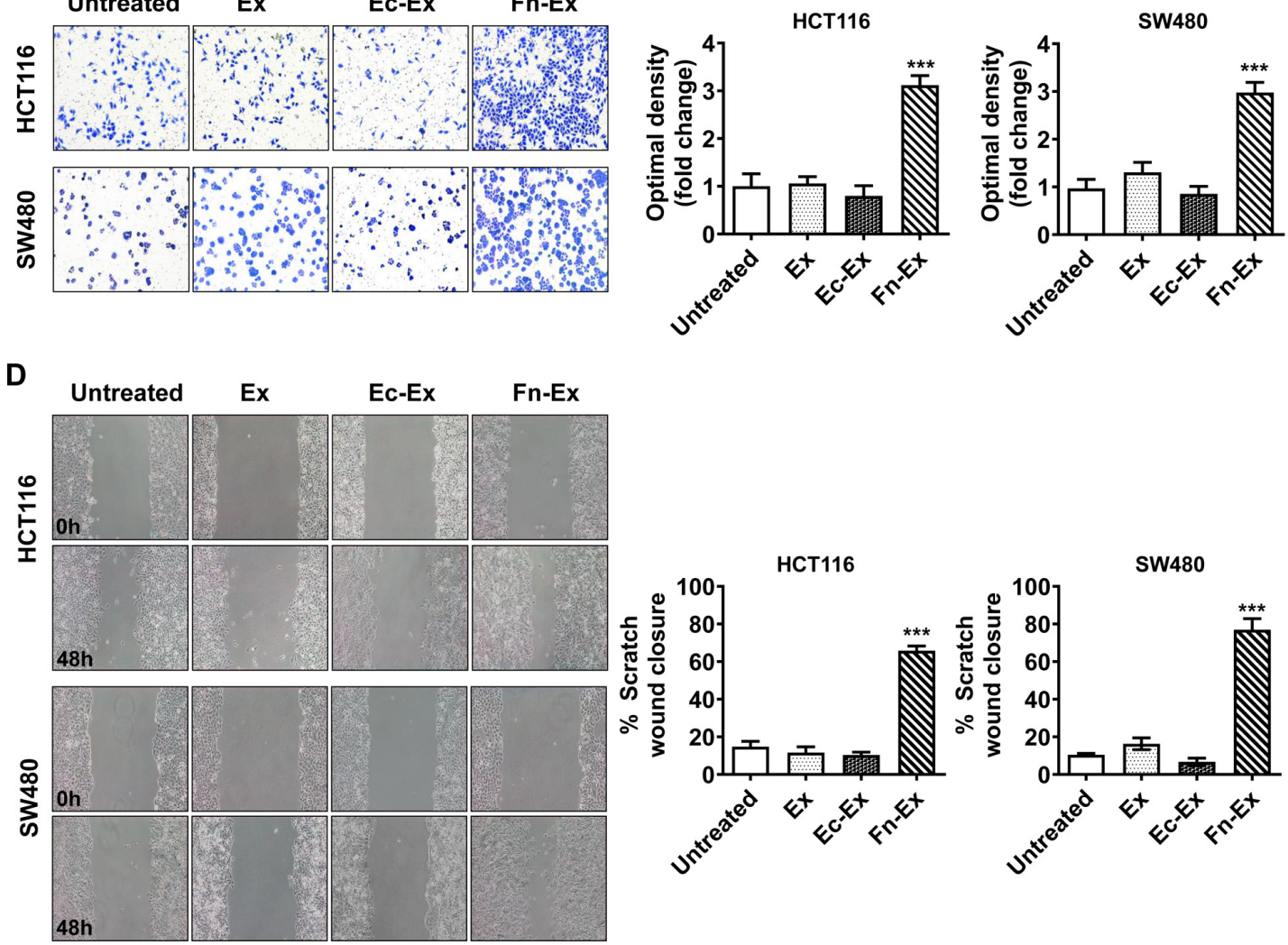

Figure 2 Fn-Ex promotes the migration of CRC cells. (A) CCK8 assays were used to detect the effects of Ex, Ec-Ex or Fn-Ex on cell proliferation at 24, 48 and 72 hours in HCT116 and SW480 cells. (B) Morphological changes in HCT116 and SW480 cells were observed following treatment with Ex, Ec-Ex or Fn-Ex for 48 hours. (C) The migration of Ex, Ec-Ex or Fn-Ex-treated CRC cells was assessed using Transwell migration assays. Representative images of the assay (right). (D) Analysis of CRC cells migration by in vitro scratch assays. Images were acquired at 0 and 48 hours. Quantitative analysis of scratch wound closure (right). Data represent at least three experiments performed in triplicate. Scale bar $=200 \mu \mathrm{m} ; \mathrm{error}$ bars, $\mathrm{SD}$ : ${ }^{*} \mathrm{p}<0.05$; $* * * p<0.001$. CRC, colorectal cancer.

of known miRNAs in Fn-Ex and Ex, respectively, were identified (online supplemental table S4). In addition, 282 miRNAs were simultaneously identified in both groups. The number of overlapping and unique miRNAs between the two groups is shown in figure $3 \mathrm{~B}$.

We next compared the expression levels of miRNAs in Fn-Ex and Ex. We found that 91 miRNAs were significantly different between the two groups $(p<0.05)$ (figure $3 \mathrm{C}$; online supplemental table S5). There were 23 miRNAs upregulated and 1 miRNA downregulated in Fn-Ex compared with Ex (normalised value $\geq 2 ; p<0.05$ ) (figure $3 D$ ). Furthermore, the top 10 most highly expressed miRNAs were chosen for validation by RT-qPCR in Fn-Ex, Ex, Ec-Ex and K-Fn-Ex (exosomes from heat-killed Fn-infected HCT116 cells). Five miRNAs (miR27a-3p, miR-21-5 p, miR-7704, miR-92b-3p and miR-1246) were confirmed to be significantly increased in Fn-Ex (all, $\mathrm{p}<0.01)$. In particular, Fn infection dramatically increased the expression levels of three miRNAs, miR-1246/92b-3p/27a-3p (miR-1246, miR-92b-3p and miR-27a-3p), as observed when comparing Fn-Ex with Ex, Ec-Ex and K-Fn-Ex (all, p<0.001) (figure 3E, online supplemental figure S3).

These data indicated that live Fn infection altered exosomal miRNA profile of CRC cells, which was accompanied with increased expression of miR-1246/92b-3p/27a-3p.

Exosomal miR-1246/92b-3p/27a-3p promotes CRC cells migration by targeting GSK3 $\beta$ and activating the Wnt/ $\beta$ catenin pathway

To explore the underlying biological functions of exosomal miRNAs during Fn infection, we chose the three miR-1246/92b$3 \mathrm{p} / 27 \mathrm{a}-3 \mathrm{p}$ generated overexpressing (mimics) and knocked 
A

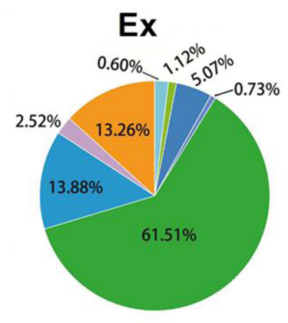

Fn-Ex

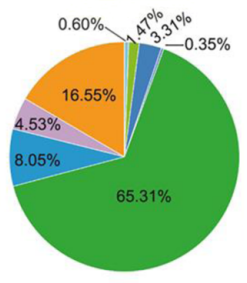

B

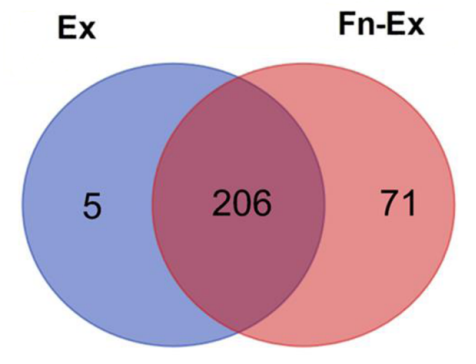

E

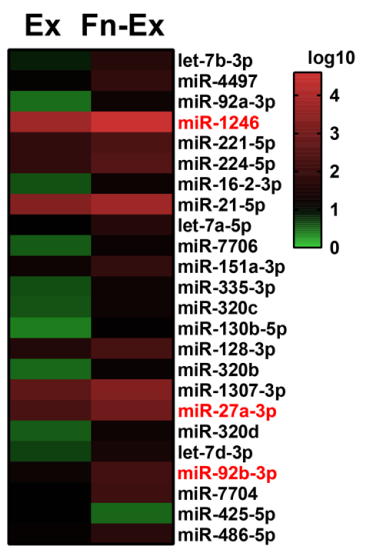

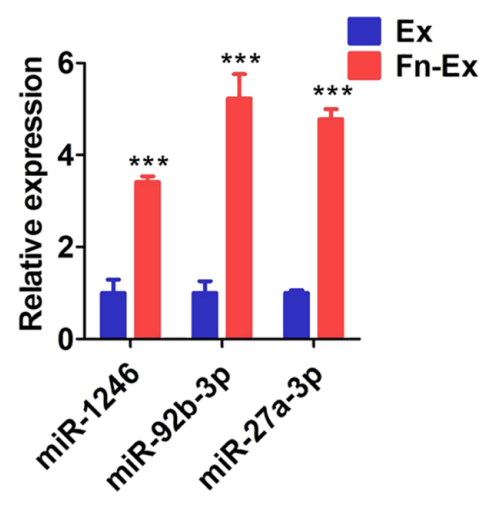

Figure 3 miR-1246/92b-3p/27a-3p upregulated in Fn-Ex. (A) The percentage of small RNA categories in all reads mapped to non-coding RNA databases. (B) Venn diagram showing the unique and overlapping miRNAs between HCT116 cells and Fn-infected HCT116 cells-derived exosomes (Ex and Fn-Ex). (C) Differentially expressed miRNAs between the Ex and Fn-Ex groups. Red, increased expression; green, decreased expression; and grey, no difference. $p<0.05$ and fold change $>2$ were considered significant. (D) Heatmap diagram of differential miRNA expression between Ex and Fn-Ex. (E) Quantitative real-time PCR validated the increase of miR-1246, miR-92b-3p and miR-27a-3p in Fn-Ex. Error bars, SD: *** $<<0.001$.

down (inhibitors) miRNAs. Transwell migration assays showed that miR-1246/92b-3p/27a-3p mimics greatly promoted migration in HCT116 cells, compared with that of the negative control (NC), while the combination of three miRNA inhibitors partially abolished Fn-Ex-mediated migration. Similar trends were also observed in SW480 cells (figure 4A). In addition, a scratch wounding assays showed that miR-1246/92b-3p/27a-3p mimics obviously promoted wound closure, compared with that of the control in HCT116 cells and SW480 cells, while the combination of three inhibitors partly rescued the Fn-Exmediated wound closure rate (figure 4B).

Next, based on the predictive results of the bioinformatics analysis, we found that miR-1246/92b-3p/27a-3p can directly target the 3'-UTR of GSK3 $\beta$ mRNA, and the association is highly conserved among species (online supplemental table S6). We further generated luciferase reporter plasmids that harboured either wild-type (WT) or mutated-type miR-1246/92b$3 p / 27 a-3 p$ binding sites within the 3'-untranslated region (UTR) of GSK3 $\beta$ (figure $5 \mathrm{~A}-\mathrm{C}$ ). As shown in figure $5 \mathrm{D}-\mathrm{F}$, miR-1246/92b-3p/27a-3p mimics and Fn-Ex or inhibitors significantly suppressed or increased luciferase activity of the reporter genes containing the WT 3'-UTR of GSK3 $\beta$ (all, $\mathrm{p}<0.05$ ), but no inhibitory effects were observed in mutated cell lines. Moreover, we found that GSK3 $\beta$ was consistently downregulated in miR-1246/92b-3p/27a-3p overexpressing cells while upregulated in miR-1246/92b-3p/27a-3p inhibited cells, suggesting that GSK3 $\beta$ was a putative target gene of miR-1246/92b-3p/27a-3p (figure $5 \mathrm{G}$ ). Furthermore, Transwell migration and woundhealing scratch assays displayed that GSK3 $\beta$ overexpression markedly retarded and GSK3 $\beta$ knockdown slightly promoted miR-1246/92b-3p/27a-3p mimics-mediated and Fn-Ex-mediated migration in both CRC cell lines (online supplemental figure S4).

Next, the expression of GSK3 $\beta$ and the Wnt $/ \beta$-catenin pathway was examined by Western blot. As shown in figure 6, GSK3 $\beta$ levels were significantly decreased in Fn-Ex treated HCT116 and SW480 cells compared with that of Ex-treated cells, while $\beta$-Catenin levels were increased in both Fn-Ex treated cell types. Moreover, decreased expression of the epithelial marker E-cadherin and increased expression of the mesenchymal marker Vimentin were observed after Fn-Ex treatment. The expression of the proto-oncogenes Cyclin D1 and C-Myc were increased in Fn-Ex treatment. Similar results were also observed in three miR-1246/92b-3p/27a-3p mimics. Collectively, these results indicated that during Fn infection, miR-1246/92b-3p/27a-3penriched exosomes induce CRC cells migration via targeting GSK3 $\beta$ and activating the $\mathrm{Wnt} / \beta$-catenin pathway.

\section{Identification of exosome proteins and functional categorisation in Fn-infected CRC cells}

To screen the specific proteins carried by Fn-Ex, the differentiated protein expression profiles of Fn-Ex and Ex were investigated by proteomic analysis. A total of 384 and 550 proteins were detected by Nano-LC-MS/MS analysis in Fn-Ex and Ex, respectively (online supplemental table S7). Venn diagram analyses revealed 197 proteins overlapping between the two groups. Fn-Ex carried 187 unique proteins, including $85 \mathrm{Fn}$ proteins and 102 cell proteins (figure 7A). Among 102 unique Fn-Ex cell 

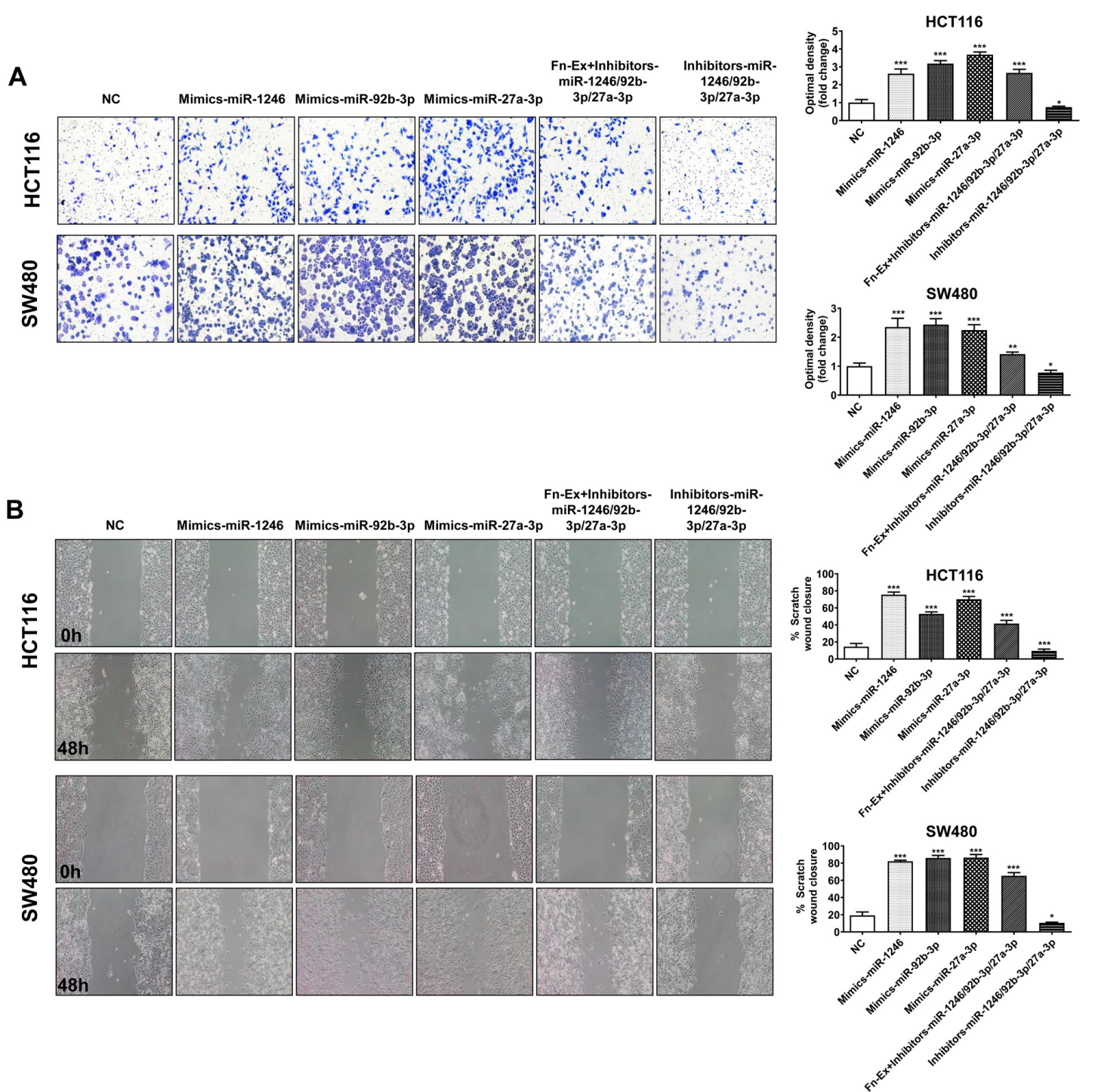

Figure 4 Exosomal miR-1246/92b-3p/27a-3p promotes the migration of CRC cells. (A) The migration of CRC cells was assessed using a Transwell migration assays. Representative images of the assay (right). (B) Analysis of CRC cells migration by in vitro scratch assays. Images were acquired at 0 and 48 hours. Right, quantitative analysis of scratch wound closure. Data represent at least three experiments performed in triplicate. Scale bar $=200 \mu \mathrm{m}$; error bars, SD: ${ }^{*} \mathrm{p}<0.05 ;{ }^{* *} \mathrm{p}<0.01 ;{ }^{* * *} \mathrm{p}<0.001$. CRC, colorectal cancer.

proteins, the most significantly enriched GO terms were structural molecule activity and molecular transducer activity (online supplemental figure S5). KEGG pathway analysis showed that the ribosome, chemokine signalling pathway and mTOR signalling pathway were enriched among these proteins (figure 7B; online supplemental table S8). It is well known that chemokine signalling pathways are involved in tumour metastasis. We further selected three proteins with chemokine signalling pathways for validation by immunoblotting. As shown in figure 7C, interleukin-8 (IL-8), C-X-C motif chemokine 16 (CXCL16) and Ras homolog family member A (RhoA) were more enriched in Fn-Ex than in Ex. Interestingly, CXCL16 exhibited the strongest abundance in Fn-Ex compared with Ex. In addition, Western blot analysis revealed that the expression of CXCL16 was increased in Fn infected HCT116 cells (figure 7D).

In addition, Transwell migration assays showed that Fn-Ex promoted migration $(2.2$-fold, $\mathrm{p}<0.01 ; 3.7$ fold, $\mathrm{p}<0.001)$ and that treatment with CXCR6 protein largely abrogated Fn-Exmediated migration (1.6 fold, $\mathrm{p}<0.05 ; 1.7$-fold, $\mathrm{p}<0.05$ ) in both HCT116 and SW480 cells, respectively (figure 7E).
Migration was also blocked by treatment with CXCR6 in the wound-healing scratch assays (average $57.9 \%$ vs $29.9 \%$, $<<0.01$; $81.6 \%$ vs $31.5 \%, \mathrm{p}<0.001$ ) (figure $7 \mathrm{~F}$ ). These data suggest that Fn infection could increase the expression of the metastasisassociated protein CXCL16 in CRC cells, and CXCL16 was further selectively packaged and enriched in Fn-Ex, promoting tumour cell migration via the CXCL16/CXCR6 axis.

\section{Exosome secreted from Fn-infected cells promote CRC metastasis}

To evaluate the potential contribution of Fn-infected exosomes to tumour metastasis in vivo, Fn-Ex activity was evaluated in two mouse colorectal tumour models. Exosomes from HCT116 cells which were transfected with miR-1246/27a-3p/92a-3p inhibitors and siRNA-CXCL16 were used as control for Fn-Ex group (si-Ex). HCT116 cells were injected subcutaneously (into $\mathrm{BALB} / \mathrm{c}$ nude mice to develop a human CRC xenograft model. Ex, Fn-Ex or si-Ex was administered intratumourally every other day once the tumour volume reached $50 \mathrm{~mm}^{3}$ (figure $8 \mathrm{~A}$ ). 
A

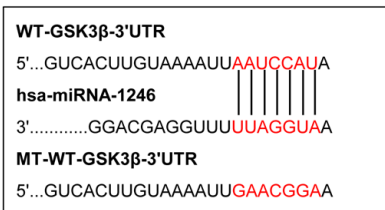

B

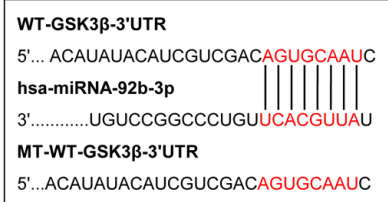

C

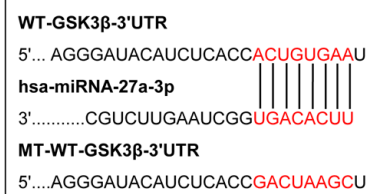

G

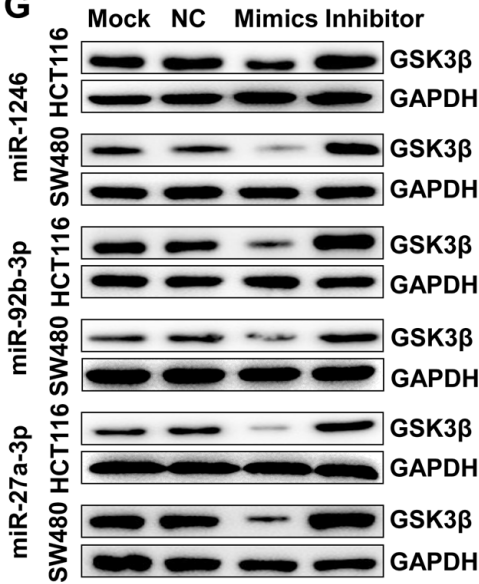

D

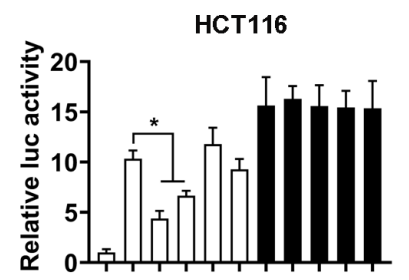

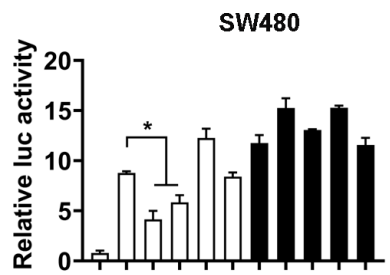
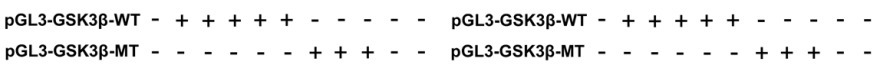

Mimics - - + - - - + - -

Fn-Ex - - + - - - + -

Inhibtor - - - + - - - +

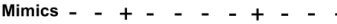

Fn-Ex - - + - - + -

Inhibtor - - - + - - - +

NC - - - $-+\cdots$

NC - - - - + - - +

E
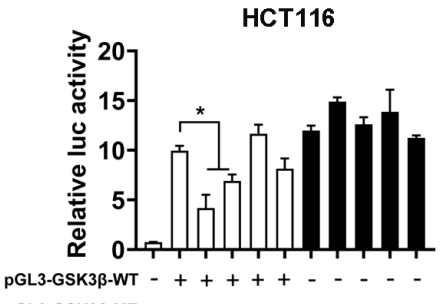

pGL3-GSK3ß-MT - - - - - - + + + -

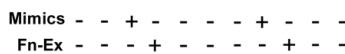

Fn-Ex - - + - - - + -

Inhibtor - - - + - - - +

NC - - - $-+\ldots$
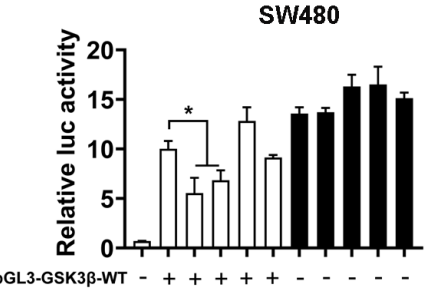

PGL3-GSK3ß-MT - - - - - $+++\cdots$

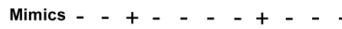

Fn-Ex - - + - - - + -

Inhibtor - - - + - - - +

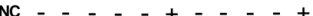

$\mathbf{F}$
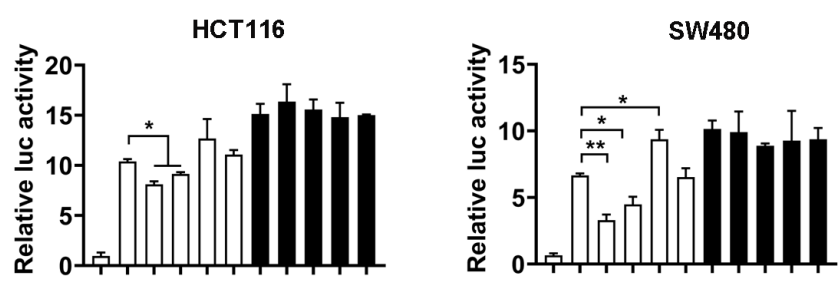

pGLS-GSK3-GSK
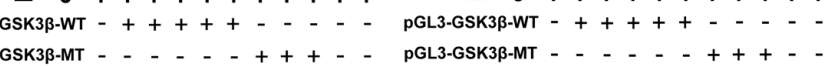

Mimics - - + - - - + -

pGL3-GSK3ß-MT - - - - - + + + -

Fn-Ex - - + - - - + -

Inhibtor - - - + - - - +

Mimics - - + - - - + - -

Fn-Ex - - + - - - + -

Inhibtor - - - + - - + -

NC - - - - + - - +

Figure 5 Identification of GSK3 $\beta$ as a direct target of miR-1246/92b-3p/27a-3p. Luciferase reporter plasmids that harbour either WT or MT miR1246 (A), miR-92b-3p (B) and miR-27a-3p (C) binding sites in the 3'-UTR of GSK3 3 . HCT116 and SW480 cells were transiently transfected with luciferase constructs along with miR-1246 (D), miR-92b-3p (E) and miR-27a-3p (F) mimics, Fn-Ex (10 $\mu \mathrm{g} / \mathrm{mL})$, inhibitors or negative controls. After 48 hours, the luciferase activity was measured. (G) Representative Western blot images showing that overexpression or inhibition of miR-1246, miR92b-3p and miR-27a-3p downregulated or upregulated GSK3 3 expression, respectively, in HCT116 cell and SW480 cells. Data represent at least three experiments performed in triplicate. Error bars, SD: ${ }^{*} p<0.05 ;{ }^{* *} p<0.01$. MT, mutated type; UTR, untranslated region; WT, wild type.

Twenty-one days later, the tumours were harvested by surgical removal (figure $8 \mathrm{~B}$ ). The results showed that mice treated with Fn-Ex did not show differences in tumour diameter, tumour volume or body weight loss from the Ex, si-Ex or PBS groups (online supplemental figure S6). However, H\&E stained liver sections showed obvious intrahepatic metastasis around blood vessels in the Fn-Ex groups, while the other three groups showed few signs of metastasis (figure 8C,D). Moreover, the expression of GSK3 $\beta$ was decreased in tumour tissues of the Fn-Ex administered group by both immunohistochemical and Western blotting analyses, whereas si-Ex significantly reversed the expression of GSK3 $\beta$ (figure 8E,F).

Additionally, we also investigated whether Fn-Ex could enhance tumour metastasis in mice with CT26 lung homografts. Ex, Fn-Ex, si-Ex or PBS control was administered intravenously (figure 8G). All four groups developed obvious tumour nodules on the pulmonary surface, and the Fn-Ex treated group presented the highest number of nodules, compared with the other three groups $(\mathrm{p}<0.001)$ (figure $8 \mathrm{H}, \mathrm{I}) . \mathrm{H} \& \mathrm{E}$ stained lung sections showed the largest area and highest number of cancer nests inside damaged lung tissues in Fn-Ex group (figure 8J). Moreover, the expression of GSK3 $\beta$ was decreased in lung tissues of the Fn-Ex administered group (figure 8K,L).

Collectively, these results suggest that exosomes from Fn-infected cells significantly facilitate tumour metastasis by carrying the cargo of Fn infection-specific miRNA and protein in vivo.

\section{Association of miR-1246/92b-3p/27a-3p and CXCL16 with} clinical characteristics of patients with CRC

We further isolated and characterised exosomes from the serum of patients with CRC and from HS. Western blots showed that these exosomes were highly enriched for the exosome-associated proteins CD63 and CD9 (figure 9A). Total RNA was further extracted from circulating exosomes. We compared the three mean values of miR-1246/92b-3p/27a-3p between samples from 

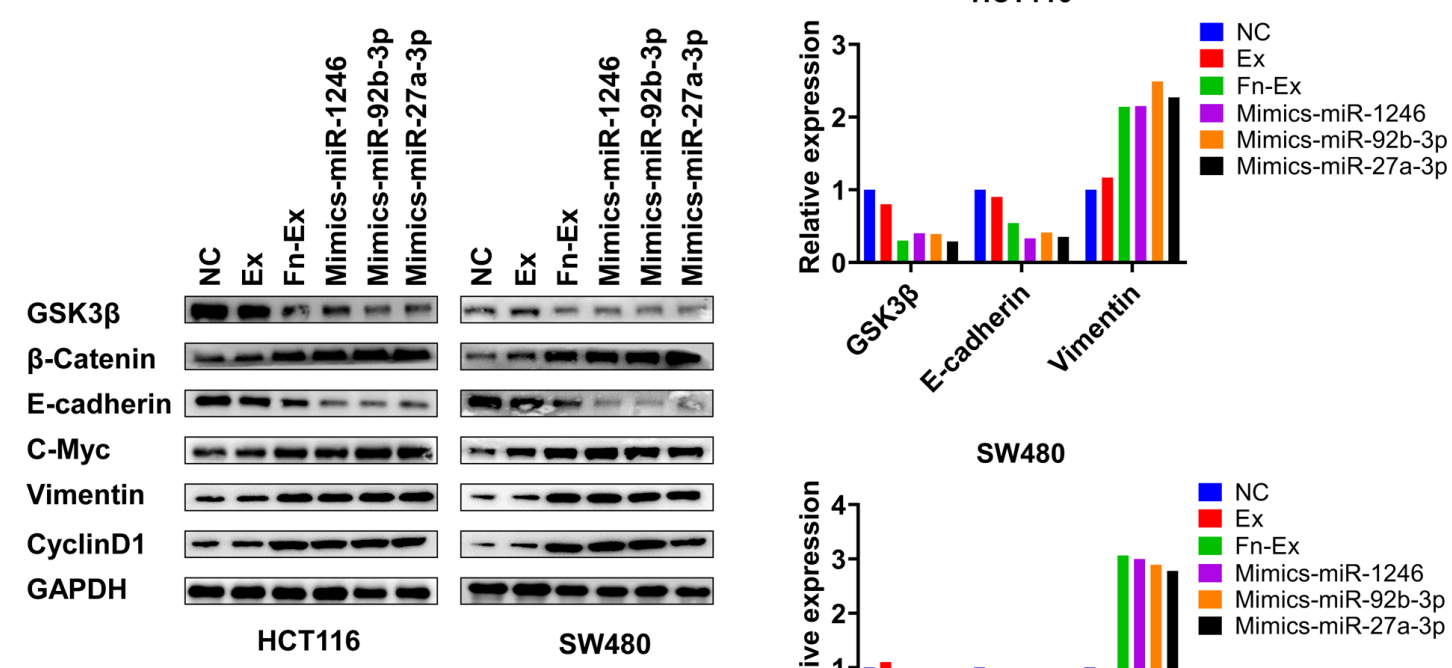

each patient. Patients with CRC $(n=40)$ had significantly higher levels of circulating exosomal miR-1246/92b-3p/27a-3p than the HS $(n=40)$ (all, $p<0.001)$ (figure 9B).

Furthermore, the carriage of Fn was investigated in faecal samples $(n=82)$ by qPCR. Pearson's correlation coefficient and linear regression analysis were applied to analyse the correlation between the levels of Fn and circulating exosomal miR-1246/92b-3p/27a-3p. Abundance of Fn showed a positive correlation with miR-1246 $(\mathrm{r}=0.232, \mathrm{p}=0.04)$ and $\operatorname{miR}-27 \mathrm{a}-3 \mathrm{p}$ $(\mathrm{r}=0.370, \mathrm{p}=0.001)$ levels, but there was no correlation with miR-92b-3p $(r=0.018, p=0.870)$ (figure 9C).

In addition, circulating exosomal CXCL16 levels were significantly upregulated in patients with CRC compared with HS $(\mathrm{p}<0.001)$ (figure 9D). More importantly, circulating exosomal CXCL16 was positively correlated with the abundance of Fn in patients with CRC $(r=0.319, p=0.03)$ (figure 9E).

Next, the correlation between circulating exosomal miR-1246/92b-3p/27a-3p, CXCL16 and clinicopathological parameters was analysed (online supplemental table S9). Circulating exosomal miR-1246 and miR-92b-3p were not obviously correlated with CRC age, sex, gender, tumour location, histological differentiation, $\mathrm{T}$ classification, $\mathrm{N}$ classification, metastasis or expression of tumour markers CEA, CA19-9 or gFOBT. However, there was a significant association between miR-27a-3p levels and clinical stage $(p=0.04)$. Further, circulating exosomal CXCL16 levels were significantly associated with metastasis $(\mathrm{p}=0.006)$.

These results indicate that the levels of circulating exosomal miR-1246, miR-27a-3p and CXCL16 were elevated and exhibit a positive correlation with gut Fn abundance in the patients with CRC, suggesting that excessive Fn levels might contribute to tumour progression through the release of Fn-associated exosomes.

\section{DISCUSSION}

Here, we found that exosomes from Fn-infected CRC cells carried both bacteria and host components that were internalised by uninfected cells. We further observed an increased size and enhanced release of exosomes from Fn-infected cells, which is in line with the features of EV from other bacterial infections such as Mycobacterial and Legionella pneumophila, ${ }^{19} 20$ indicating that Fn stimulated the production of exosomes during infection.

Currently, numerous studies have reported that exosomes isolated from cells infected with various intracellular pathogens contain microbial components. ${ }^{2122}$ However, almost all of these studies have focused on the role of exosomes in infection and immunity, which few have focused on the role of cancer progression. It has been well recognised that $\mathrm{Fn}$ is a powerful driving force for CRC progression. ${ }^{15}$ Moreover, as an important intratumour bacterium, Fn participates in tumour progression and mediates tumour resistance to chemotherapeutic drugs. ${ }^{23}$ Most excitingly, our study reveals that exosomes from Fn-infected cells play a newly identified role promoting cancer progression.

miRNAs have recently emerged as major players in the interactions between host cells and bacterial pathogens. Substantial evidence confirms that infections with pathogenic organisms lead to significant changes in exosomal miRNA. Our study found Fn infection alters exosomal miRNA profiles of CRC cells. Usually, bacteria modify host miRNA expression for their own benefit, promoting survival, replication and persistence. ${ }^{24}$ Similarly, exosome-mediated transfer of miRNA to neighbouring cancer cells might help Fn to survival inside tumour tissues.

Furthermore, many studies have demonstrated that miRNAs play pathogenic role in the development of colitisassociated colon cancer. Some data have highlighted that specific miRNAs contribute to colorectal carcinogenesis, and several such miRNAs can serve as biomarkers for diagnosis, prognosis, and metastasis prediction in patients with CRC. ${ }^{25}$ We confirmed that metastasis-related miRNAs, including 
A

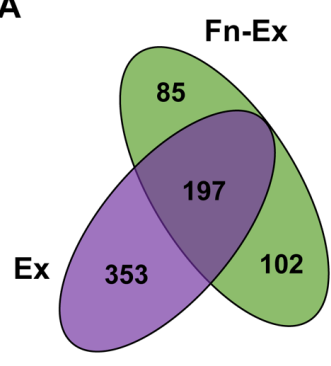

B

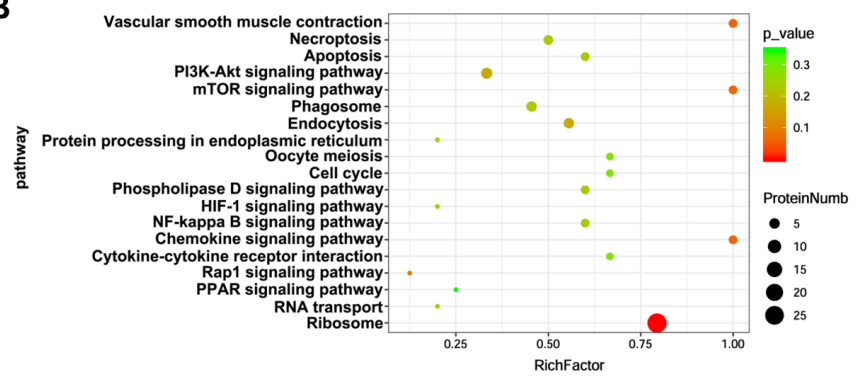

D

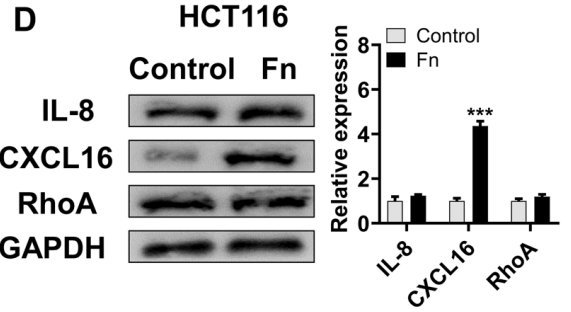

HCT116

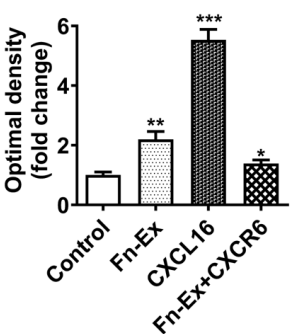

SW480

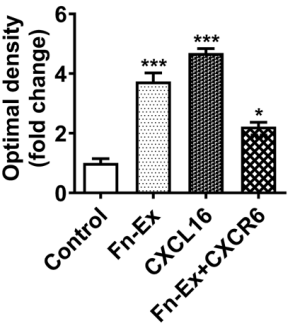

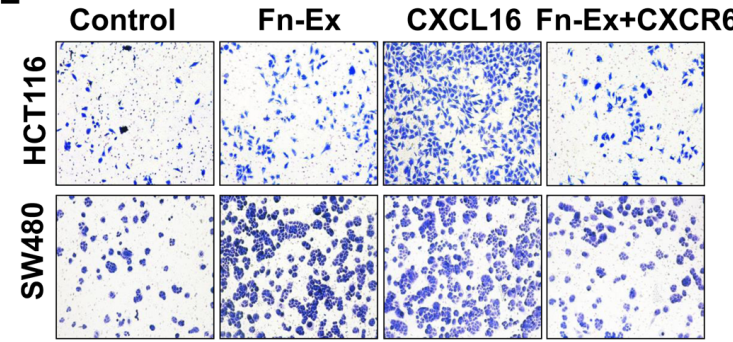

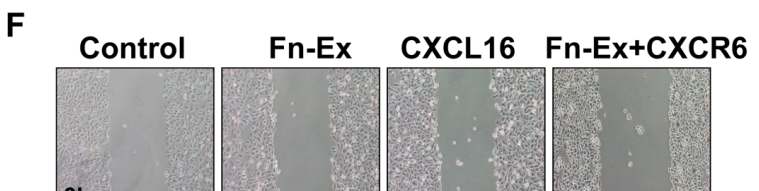

HCT116
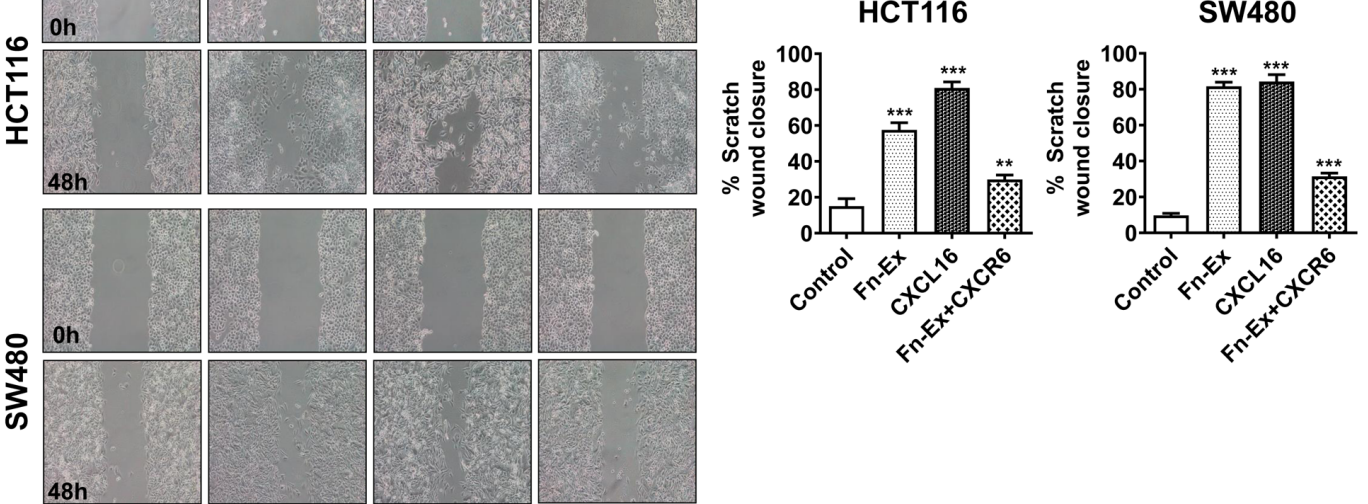

Figure 7 CXCL16 of Fn-Ex to promote CRC cells migration in vivo. (A) Venn diagram showing the unique and overlapping proteins between HCT116 cells and Fn-infected HCT116 cells-derived exosomes (Ex and Fn-Ex). (B) KEGG pathway analysis of Fn-infected HCT116 cells-derived exosome (Fn-Ex) unique cell proteins. (C) The protein levels of IL-8, CXCL16 and RhoA were detected by Western blot in HCT116 cells and Fn-infected HCT116 cellsderived exosomes (Ex and Fn-Ex). Right, quantitatively analysed. (D) The protein levels of IL-8, CXCL16 and RhoA were detected by Western blot in HCT116 cells. On the right, the quantitatively analysis. Migration of HCT116 cells and SW480 cells that were untreated, or treated with Fn-Ex, CXCL16 or Fn-Ex $+C X C R 6$, as detected by Transwell migration assays (E) and wound healing assays (F). Data represent at least three experiments performed in triplicate. Scale bar $=200 \mu \mathrm{m}$; error bars, SD: ${ }^{*} p<0.05 ;{ }^{* *} p<0.01 ;{ }^{* *} p<0.001$. CRC, colorectal cancer.

miR-1246, miR-92b-3p and miR-27a-3p, were selectively secreted in Fn-Ex and enhanced CRC migration. Consistent with our research, CRC cells-derived exosomes containing miR-1246 were reported to promote angiogenesis. ${ }^{26}$ Moreover, both miR-92b-3p and miR-27a-3p were reported to promote CRC cells proliferation, invasion and migration, ${ }^{27-29}$ and the serum exosomal levels of miR-1246 and miR-27a-3p were validated as biomarkers of colon cancer. ${ }^{3031}$

An increasing number of studies have identified specific miRNAs (eg, miR-21, miR-155 and miR-103a) that lead to 
A

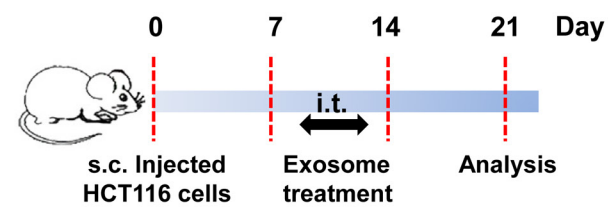

C

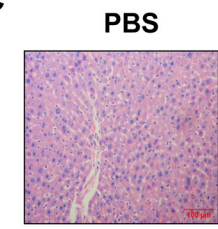

D

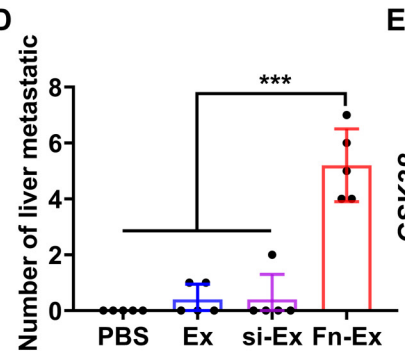

F

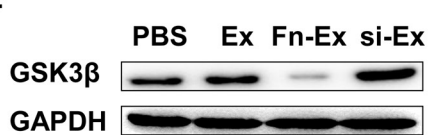

G
B

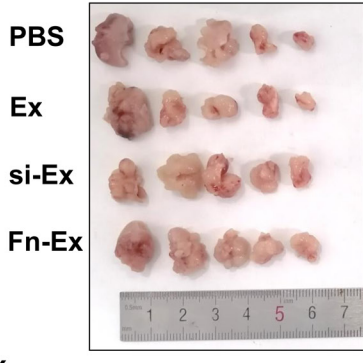

Fn-Ex

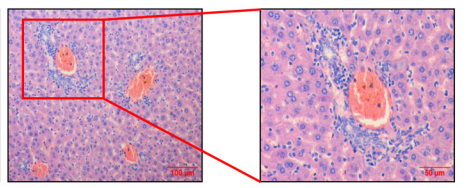

E

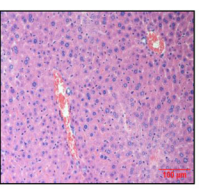

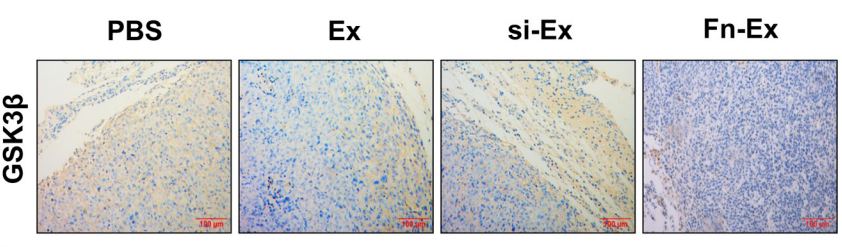

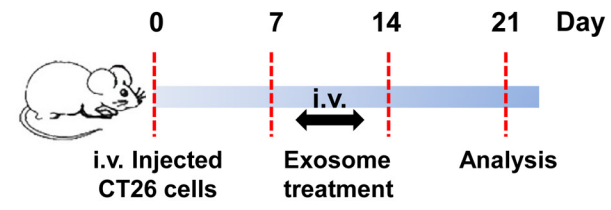

H

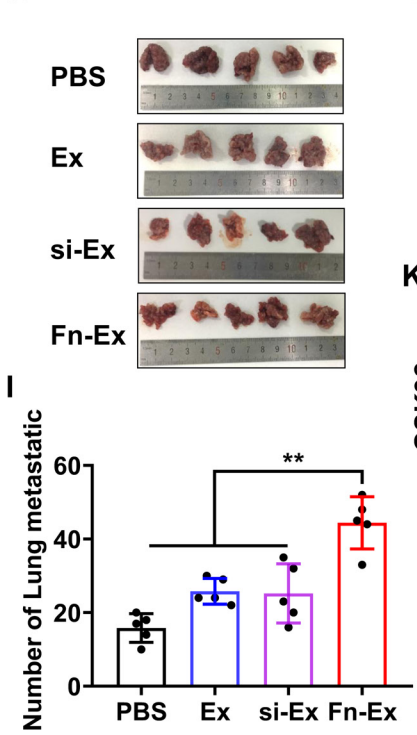

J

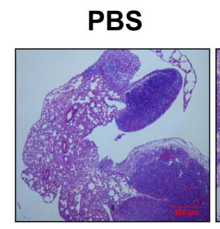

Ex

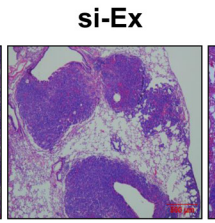

Fn-Ex

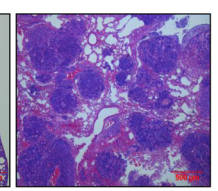

K
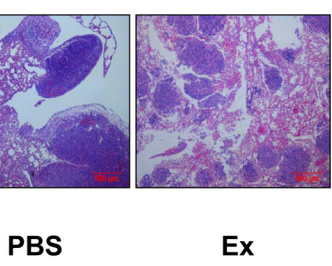

Ex

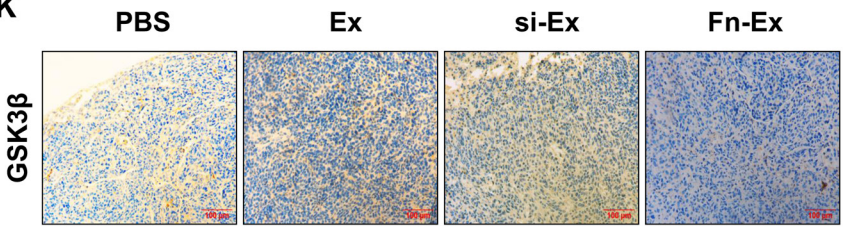

Fn-Ex

L

PBS Ex Fn-Ex si-Ex

GSK3 $\beta$

GAPDH

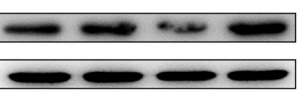

Figure 8 In vivo verification shows that Fn-Ex promotes migration. (A,B) A flowchart depicting the in vivo experimental design and morphology. (C) Histopathological examination of the liver tissue sections $(n=5)$ and $(D)$ the number of liver tumours were quantitatively analysed. (E) Immunohistochemical analysis of the paraffin-embedded tumour tissues using a GSK3 $\beta$ antibody $(n=5)$. ( $F)$ GSK3 $\beta$ expression in implanted tumours $(n=5)$. (G) A flowchart depicting the in vivo experimental design. (H) Photograph of the harvested lungs showing regions with multiple nodules ( $n=5$ ) and (I) quantitative analysis. (J) Histopathological examination of the lung tissue sections $(n=5)$. (K) Immunohistochemical analysis of the paraffinembedded lung tissues using a GSK3 $\beta$ antibody $(n=5)$. (L) GSK3 $\beta$ expression in implanted tumours $(n=5)$. Error bars, SD: ${ }^{* *} p<0.01 ;{ }^{* * *} p<0.001$.

alteration of $\mathrm{Wnt} / \beta$-catenin signalling in CRC. ${ }^{32}$ Moreover, recent evidence has suggested that premetastatic niches in the liver could be promoted by miR-21 in exosomes from CRC cells through the miR-21-TLR7-IL6 axis. ${ }^{33}$ Significantly increased levels of serum exosomal miR-6803-5 $\mathrm{p}$, miR-17-5 $\mathrm{p}$ and miR$92 \mathrm{a}-3 \mathrm{p}^{34} 35$ and decreased levels of miR-548c-5p and miR-638 have been recently associated with poor survival and liver metastasis in patients with CRC. ${ }^{36}{ }^{37}$ In line with these studies, 
A

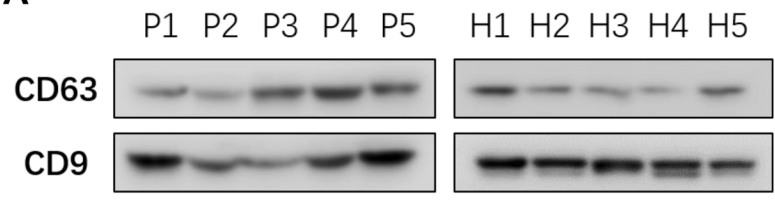

B
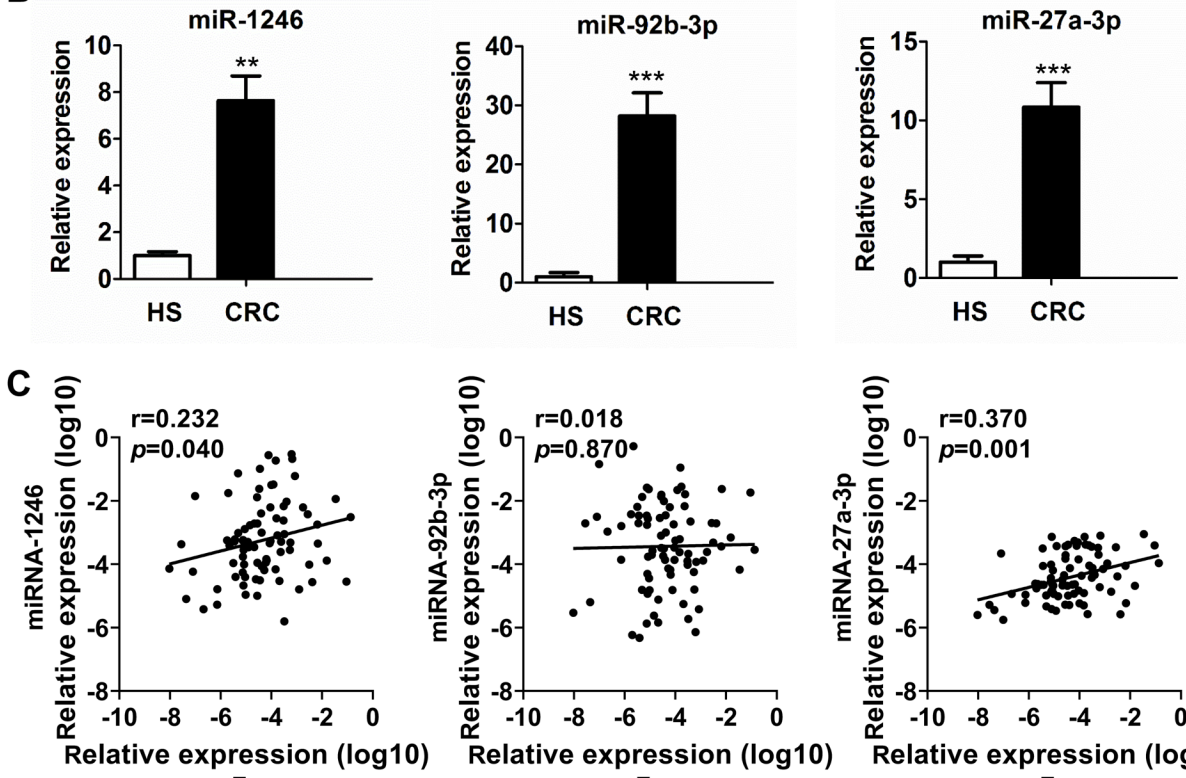

$\mathrm{Fn}$
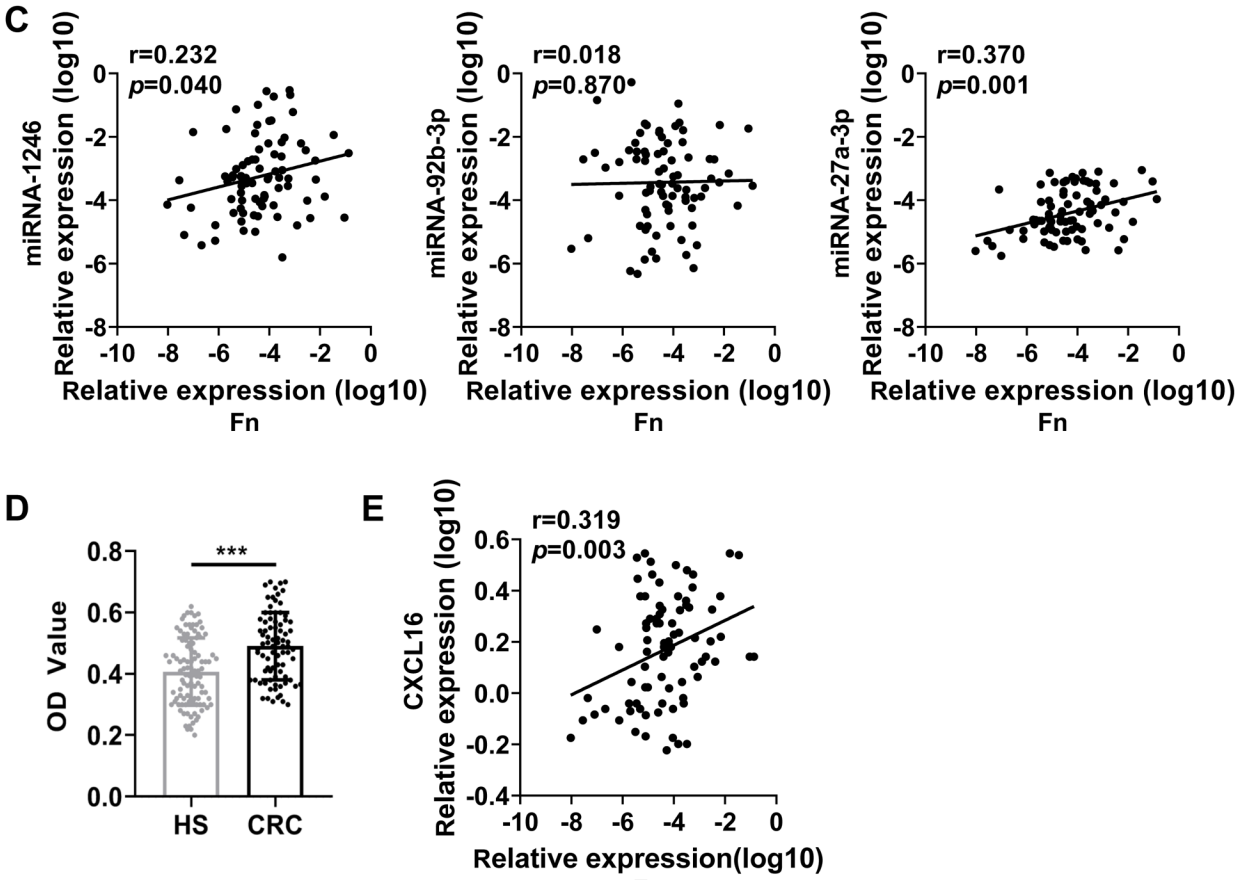

Fn

Figure 9 Circulating exosomal miR-1246/92b-3p/27a-3p and CXCL16 levels correlate with Fn in patients with CRC. (A) Western blot analysis of the expression of markers (CD63 and CD9) in serum exosomal protein purified from healthy subjects (H1-H5) and patients with CRC (P1-P5). (B) Exosome miR-1246, miR-92b-3p and miR-27a-3p levels were measured by RT-qPCR in the serum of patients with CRC and HS. (C) Correlation between the abundance of $\mathrm{Fn}$ in the faeces of patients with CRC and the levels of miR-1246, miR-92b-3p and miR-27a-3p in exosomes from the serum of patients with CRC. Patients were analysed by Pearson's correlation coefficient and linear regression. (D) Exosome CXCL16 levels were measured by ELISA in the serum of patients with CRC and HS. (E) Correlation between the abundance of Fn in faeces and the levels of CXCL16 in circulating exosomes from patients with CRC. Error bars, SD: ${ }^{* *} \mathrm{p}<0.01 ;{ }^{* *} \mathrm{p}<0.001$. CRC, colorectal cancer; HS, healthy subjects.

we identified that metastasis-related exosomes can be transported from Fn-infected CRC cells to non-infected cells. These miR-1246 ${ }^{+}$miR-92b-3p ${ }^{+}$miR-27a-3p ${ }^{+}$exosomes modulated the bioactivity of uninfected cells by activating $\mathrm{Wnt} / \beta$-catenin signalling and downregulating GSK3 $\beta$.

Usually, exosomes from bacteria-infected hosts can induce the biosynthesis of various cytokines in recipient cells. ${ }^{38}$ Exosomes from Salmonella-infected macrophages increased TNF- $\alpha$ production by human monocytes. ${ }^{38}$ Exosomes from Mycoplasma-infected cells induce a mixed cytokine response, including the production of both IFN- $\gamma$ and IL-10 from B cells. Exosomes from Mycobacterialinfected cells secreted TNF- $\alpha$ and IFN- $\gamma$. These cytokines promote both recruitment and activation of immune cells and may play a role in promoting the innate immune response on bacterial infection.

Interestingly, in contrast to these inflammatory bacteria, we found that the cancer-causing bacterium Fn stimulated the production of the chemokines CXCL16. It is well known that chemokines and their receptors selective mediators of leucocyte migration to inflammatory sites. They also play a critical role in tumour initiation, promotion and progression. ${ }^{39}$ Numerous studies have proposed an important role for the CXCL16/CXCR6 chemokine axis in the metastasis of different tumours including prostate, liver, ovarian cancers, lung and breast cancers as well as meningioma and hepatocellular carcinoma. ${ }^{40-43}$ Specifically, elevated levels of CXCL16 were reported in the serum, tumour tissues and lymph 


\section{Fusobacterium nucleatum (Fn)}

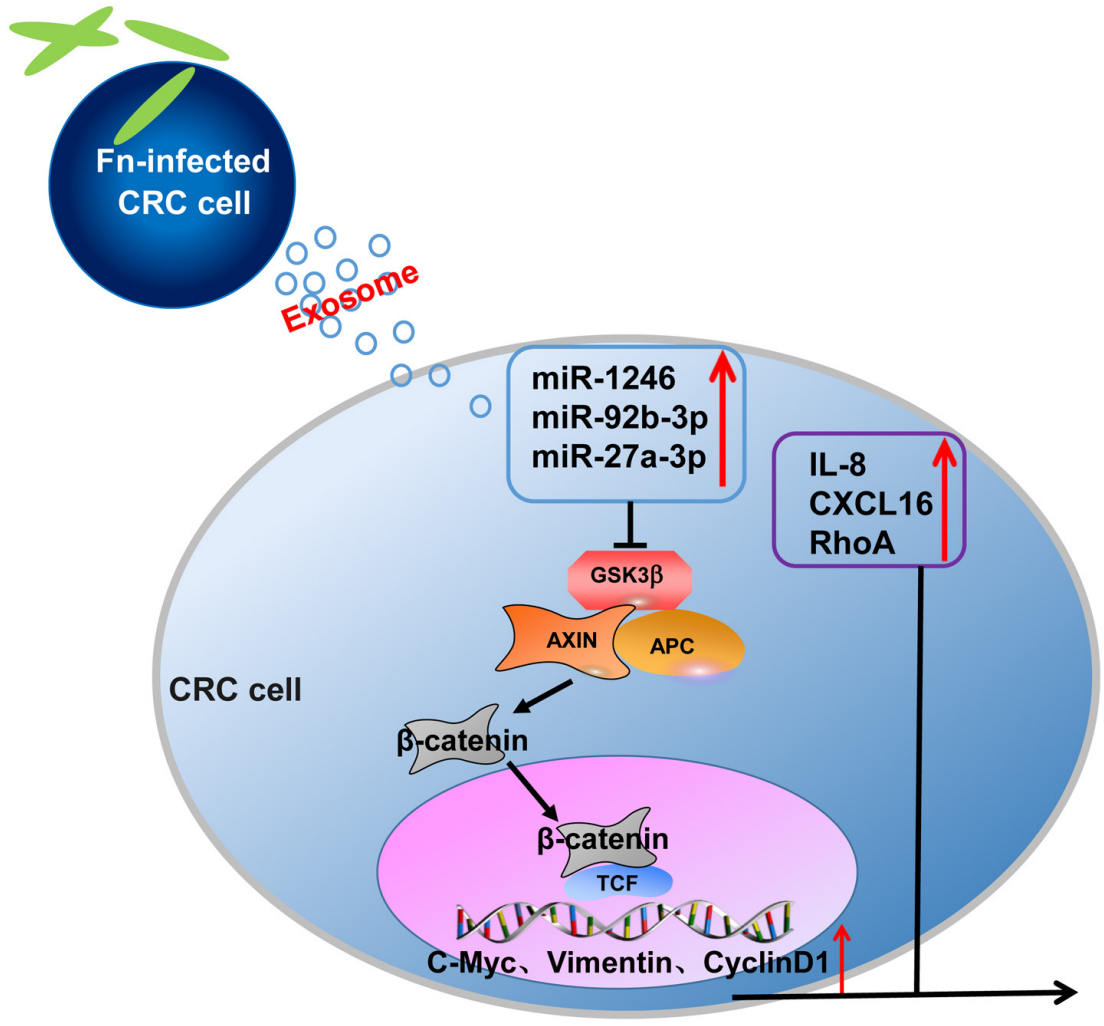

nodes of patients with CRC and indicate a sign of a poor prognosis in CRC. ${ }^{44-46}$ These observations raise the possibility that CXCL16/ CXCR6 interactions may be important for CRC invasion and metastasis. $^{46}$

Intriguingly, several groups indicated that CXCL16/CXCR6 signalling correlates with liver-specific homing and lung-specific homing during inflammatory events. ${ }^{47} 48 \mathrm{CXCR}^{+}$natural killer $\mathrm{T}$ cells are highly enriched in the human liver and are specifically recruited by liver sinusoidal endothelial cells, which are the major source of CXCL16. CCR6 has been previously associated with colorectal liver metastasis in patients with CRC. ${ }^{49}$ In our study, Fn infection induced the expression of CXCL16 in CRC cells and also promoted the release of CXCL16 loaded exosomes from these infected cells. These CXCL16 ${ }^{+}$Fn-Ex facilitate the homing of noninfected recipient CRC cells to hepatic vessels by the CXCL16/ CXCR6 axis. In light of the evidence that CXCL16 plays a role in angiogenesis, intrahepatic metastatic nests were observed to locate around blood vessels in our study. Recently, a study reported that Clostridium species modified bile acids to signal liver sinusoidal endothelial cells to produce CXCL16. ${ }^{50}$ These results that some intestinal anaerobes have the ability to stimulate the production of CXCLl6 the homing of cells to the liver.

Moreover, our proteomic analysis rapidly identified the presence of CXCL16, IL-8 and RhoA in Fn-Ex, clearly indicating their involvement in tumour invasion. Interestingly, the CXCL16/ CXCR6 axis has been reported to enhance the activity of the ERK1/2 pathway, which activates RhoA to promote tumour progression. ${ }^{41}$ Plasma levels of IL-8 have been shown to be significantly and persistently elevated after minimally invasive colorectal resection. ${ }^{51}$ Elevated CXCL16, IL-8 and RhoA were also observed in Fn-Ex in our study, suggesting that the CXCL16 ${ }^{+} \mathrm{RhoA}^{+} \mathrm{IL}-8^{+}$ exosome paly role facilitating metastasis.
TEXs are an important component of the tumour microenvironment and are currently considered to be one of the main contributors to tumour metastasis by transporting specific proteins and RNA. The specific molecules present in TEXs have been reported to determine organ-specific metastasis and the survival of patients with cancer. ${ }^{52}$ Previous data exploring the characteristics of the metastatic pattern of CRC have revealed that the liver is the main metastatic site of CRC, while lungs, bones and the brain are less common. ${ }^{53}$ Consistent with these studies, our results confirmed that Fn infection further accelerated liver metastasis by the combination of enhanced cytokines, chemokines and miRNAs via exosome release.

In summary, we elucidated a schematic model of Fn-Ex functions in tumour metastasis (figure 10). This figure depicts that Fn-infected CRC cells release exosomes carrying metastasis-related miRNAs and proteins. These Fn exosomes are internalised and drive uninfected recipient cells towards a prometastatic phenotype. We therefore conclude that TEXs released from Fn-infected tumour cells may function as messengers to promote CRC cell metastasis. Exploring the mechanism and function of Fn infectedderived exosomes may prove beneficial for preventing metastasis, and eliminating Fn infection may be a valuable therapy.

Correction notice This article has been corrected since it published Onine First. The first affiliations has been updated and a sentence within the 'Identification of exosome proteins and functional categorisation in Fn-infected CRC cells' paragraph has been amended.

Contributors SG, WLL, QZ and GZ: acquisition of data, analysis and interpretation of data, statistical analysis and drafting of the manuscript. SG, JC and FC: technical and material support. WLL and GZ: study concept and design, analysis and interpretation of data, drafting of the manuscript, obtained funding and study supervision. All authors read and approved the final manuscript. 
Funding This work was supported by the National Natural Science Foundation of China (No. 81673005; No. 81972289)

Competing interests None declared.

Patient consent for publication Not required.

Provenance and peer review Not commissioned; externally peer reviewed.

Data availability statement All data relevant to the study are included in the article or uploaded as supplementary information. ManuscriptFigureSupplementary File.

Supplemental material This content has been supplied by the author(s). It has not been vetted by BMJ Publishing Group Limited (BMJ) and may not have been peer-reviewed. Any opinions or recommendations discussed are solely those of the author(s) and are not endorsed by BMJ. BMJ disclaims all liability and responsibility arising from any reliance placed on the content. Where the content includes any translated material, BMJ does not warrant the accuracy and reliability of the translations (including but not limited to local regulations, clinical guidelines, terminology, drug names and drug dosages), and is not responsible for any error and/or omissions arising from translation and adaptation or otherwise.

ORCID iD

Ge Zhang http://orcid.org/0000-0003-3292-1114

\section{REFERENCES}

1 Théry C, Zitvogel L, Amigorena S. Exosomes: composition, biogenesis and function. Nat Rev Immunol 2002;2:569-79.

2 Colombo M, Raposo G, Théry C. Biogenesis, secretion, and intercellular interactions of exosomes and other extracellular vesicles. Annu Rev Cell Dev Biol 2014;30:255-89.

3 Tkach M, Théry C. Communication by extracellular vesicles: where we are and where we need to go. Cell 2016;164:1226-32.

4 Domenis R, Cifù A, Marinò D, et al. Toll-Like receptor-4 activation boosts the immunosuppressive properties of tumor cells-derived exosomes. Sci Rep 2019;9:8457.

5 Azmi AS, Bao B, Sarkar FH. Exosomes in cancer development, metastasis, and drug resistance: a comprehensive review. Cancer Metastasis Rev 2013;32:623-42.

6 Hoshino A, Costa-Silva B, Shen T-L, et al. Tumour exosome integrins determine organotropic metastasis. Nature 2015;527:329-35.

7 Rana S, Malinowska K, Zöller M. Exosomal tumor microRNA modulates premetastatic organ cells. Neoplasia 2013;15:281-IN31.

8 Schorey JS, Cheng Y, Singh PP, et al. Exosomes and other extracellular vesicles in hostpathogen interactions. EMBO Rep 2015;16:24-43.

9 Sundaramurthy V, Korf $\mathrm{H}$, Singla A, et al. Survival of Mycobacterium tuberculosis and Mycobacterium bovis BCG in lysosomes in vivo. Microbes Infect 2017;19:515-26.

10 Yang S, Xia Y-P, Luo X-Y, et al. Exosomal CagA derived from Helicobacter pyloriinfected gastric epithelial cells induces macrophage foam cell formation and promotes atherosclerosis. J Mol Cell Cardiol 2019;135:40-51.

11 Brennan CA, Garrett WS. Fusobacterium nucleatum - symbiont, opportunist and oncobacterium. Nat Rev Microbiol 2019;17:156-66.

12 Bullman S, Pedamallu CS, Sicinska E, et al. Analysis of Fusobacterium persistence and antibiotic response in colorectal cancer. Science 2017;358:1443-8.

13 Hendrickson EL, Wang T, Beck DAC, et al. Proteomics of Fusobacterium nucleatum within a model developing oral microbial community. Microbiologyopen 2014;3:729-51.

14 Yang Y, Weng W, Peng J, et al. Fusobacterium nucleatum increases proliferation of colorectal cancer cells and tumor development in mice by activating Toll-like receptor 4 signaling to nuclear factor- $\mathrm{KB}$, and up-regulating expression of microRNA-21. Gastroenterology 2017;152:e24:851-66.

15 Gholizadeh P, Eslami H, Kafil HS. Carcinogenesis mechanisms of Fusobacterium nucleatum. Biomed Pharmacother 2017;89:918-25.

16 Xue Y, Xiao H, Guo S, et al. Indoleamine 2,3-dioxygenase expression regulates the survival and proliferation of Fusobacterium nucleatum in THP-1-derived macrophages. Cell Death Dis 2018;9:355.

17 Wang H-F, Li L-F, Guo S-H, et al. Evaluation of antibody level against Fusobacterium nucleatum in the serological diagnosis of colorectal cancer. Sci Rep 2016;6:33440.

18 Guo S, Li L, Xu B, et al. A Simple and Novel Fecal Biomarker for Colorectal Cancer: Ratio of Fusobacterium Nucleatum to Probiotics Populations, Based on Their Antagonistic Effect. Clin Chem 2018;64:1327-37.

19 Bhatnagar S, Shinagawa K, Castellino FJ, et al. Exosomes released from macrophages infected with intracellular pathogens stimulate a proinflammatory response in vitro and in vivo. Blood 2007;110:3234-44.

20 Jung AL, Herkt CE, Schulz C, et al. Legionella pneumophila infection activates bystander cells differentially by bacterial and host cell vesicles. Sci Rep 2017;7:6301.

21 Radomski N, Karger A, Franzke K, et al. Chlamydia psittaci-Infected Dendritic Cells Communicate with NK Cells via Exosomes To Activate Antibacterial Immunity. Infect Immun 2019;88:IAI.00541-19.
22 Li H, Chi X, Li R, et al. Hiv-1-Infected cell-derived exosomes promote the growth and progression of cervical cancer. Int J Biol Sci 2019;15:2438-47.

23 Yu T, Guo F, Yu Y, et al. Fusobacterium nucleatum promotes chemoresistance to colorectal cancer by modulating autophagy. Cell 2017;170:e16:548-63.

24 Aguilar C, Mano M, Eulalio A. Micrornas at the Host-Bacteria interface: host defense or bacterial offense. Trends Microbiol 2019;27:206-18.

25 Min L, Zhu S, Chen L, et al. Evaluation of circulating small extracellular vesicles derived miRNAs as biomarkers of early colon cancer: a comparison with plasma total miRNAs. J Extracell Vesicles 2019;8:1643670.

26 Yamada N, Tsujimura N, Kumazaki M, et al. Colorectal cancer cell-derived microvesicles containing microRNA-1246 promote angiogenesis by activating Smad 1/5/8 signaling elicited by PML down-regulation in endothelial cells. Biochim Biophys Acta 2014;1839:1256-72.

27 Gong L, Ren M, Lv Z, et al. miR-92b-3p promotes colorectal carcinoma cell proliferation, invasion, and migration by inhibiting FBXW7 in vitro and in vivo. DNA Cell Biol 2018;37:501-11.

28 Su C, Huang D-P, Liu J-W, et al. miR-27a-3p regulates proliferation and apoptosis of colon cancer cells by potentially targeting BTG1. Oncol Lett 2019;18:2825-34.

29 Liang J, Tang J, Shi H, et al. miR-27a-3p targeting RXR $\alpha$ promotes colorectal cancer progression by activating Wnt/ $\beta$-catenin pathway. Oncotarget 2017;8:82991-3008.

30 Ogata-Kawata $\mathrm{H}$, Izumiya M, Kurioka D, et al. Circulating exosomal microRNAs as biomarkers of colon cancer. PLoS One 2014;9:e92921.

31 Ostenfeld MS, Jensen SG, Jeppesen DK, et al. miRNA profiling of circulating EpCAM(+) extracellular vesicles: promising biomarkers of colorectal cancer. J Extracell Vesicles 2016;5:31488.

32 Balacescu O, Sur D, Cainap C, et al. The impact of miRNA in colorectal cancer progression and its liver metastases. Int J Mol Sci 2018;19:ijms19123711.

33 Shao Y, Chen T, Zheng X, et al. Colorectal cancer-derived small extracellular vesicles establish an inflammatory premetastatic niche in liver metastasis. Carcinogenesis 2018;39:1368-79.

34 Fu F, Jiang W, Zhou L, et al. Circulating exosomal miR-17-5p and miR-92a-3p predict pathologic stage and grade of colorectal cancer. Trans/ Oncol 2018;11:221-32.

35 Yan S, Jiang Y, Liang C, et al. Exosomal miR-6803-5p as potential diagnostic and prognostic marker in colorectal cancer. J Cell Biochem 2018;119:4113-9.

36 Peng Z-Y, Gu R-H, Yan B. Downregulation of exosome-encapsulated miR-548c-5p is associated with poor prognosis in colorectal cancer. J Cell Biochem 2018:jcb.27291.

37 Yan S, Han B, Gao S, et al. Exosome-encapsulated microRNAs as circulating biomarkers for colorectal cancer. Oncotarget 2017;8:60149-58.

38 Zhang W, Jiang $\mathrm{X}$, Bao J, et al. Exosomes in pathogen infections: a bridge to deliver molecules and link functions. Front Immunol 2018;9:90.

39 Marcuzzi E, Angioni R, Molon B, et al. Chemokines and chemokine receptors: orchestrating tumor metastasization. Int J Mol Sci 2018;20:ijms20010096.

$40 \mathrm{Ke} \mathrm{C}$, Ren Y, Lv L, et al. Association between CXCL16/CXCR6 expression and the clinicopathological features of patients with non-small cell lung cancer. Oncol Lett 2017;13:4661-8

41 Xiao G, Wang X, Wang J, et al. CXCL16/CXCR6 chemokine signaling mediates breast cancer progression by pERK1/2-dependent mechanisms. Oncotarget 2015;6:14165-78.

42 Hattermann K, Bartsch K, Gebhardt HH, et al. "Inverse signaling" of the transmembrane chemokine CXCL16 contributes to proliferative and anti-apoptotic effects in cultured human meningioma cells. Cell Commun Signal 2016;14:26.

43 Liu J, Chen S, Wang W, et al. Cancer-Associated fibroblasts promote hepatocellular carcinoma metastasis through chemokine-activated hedgehog and TGF- $\beta$ pathways. Cancer Lett 2016;379:49-59.

44 Deng L, Chen N, Li Y, et al. CXCR6/CXCL16 functions as a regulator in metastasis and progression of cancer. Biochim Biophys Acta 2010;1806:42-9.

45 AbdelMageed M, Ali H, Olsson L, et al. The chemokine CXCL16 is a new biomarker for lymph node analysis of colon cancer outcome. Int J Mol Sci 2019;20:ijms20225793.

46 Chen Z, Dai W, Yang L, et al. Elevated expression of CXCL16 correlates with poor prognosis in patients with colorectal cancer. Cancer Manag Res 2019;11:4691-7.

47 Ruth JH, Haas CS, Park CC, et al. CXCL16-mediated cell recruitment to rheumatoid arthritis synovial tissue and murine lymph nodes is dependent upon the MAPK pathway. Arthritis Rheum 2006;54:765-78.

48 Agostini C, Cabrelle A, Calabrese F, et al. Role for CXCR6 and its ligand CXCL16 in the pathogenesis of T-cell alveolitis in sarcoidosis. Am J Respir Crit Care Med 2005; 172:1290-8.

49 Ghadjar P, Coupland SE, Na I-K, et al. Chemokine receptor CCR6 expression level and liver metastases in colorectal cancer. J Clin Oncol 2006;24:1910-6.

$50 \mathrm{Ma}$ C, Han M, Heinrich B, et al. Gut microbiome-mediated bile acid metabolism regulates liver cancer via NKT cells. Science 2018;360:aan5931.

51 Shantha Kumara HMC, Sutton E, Bellini GA, et al. Plasma interleukin-8 levels are persistently elevated for 1 month after minimally invasive colorectal resection for colorectal cancer. Mol Clin Oncol 2018;8:471-6.

52 Whiteside TL. Tumor-Derived exosomes and their role in cancer progression. Adv Clin Chem 2016;74:103-41.

53 Hugen $\mathrm{N}$, van de Velde $\mathrm{CJH}$, de Wilt JHW, et al. Metastatic pattern in colorectal cancer is strongly influenced by histological subtype. Ann Oncol 2014;25:651-7. 
Correction: Exosomes derived from Fusobacterium nucleatuminfected colorectal cancer cells facilitate tumour metastasis by selectively carrying miR-1246/92b-3p/27a-3p and CXCL16

Guo S, Chen J, Chen F, et al. Exosomes derived from Fusobacterium nucleatum-infected colorectal cancer cells facilitate tumour metastasis by selectively carrying miR-1246/92b3p/27a-3p and CXCL16. Gut 2021;70:1507-19. doi: 10.1136/gutjnl-2020-321187

The representative image of Ex SW480 cells in Figure 2D, the representative images of mimicsmiR-27a-3p SW480 cells in Figure 4A, and Figure 8F, L were incorrect.

The correct figures 2, 4 and 8 are shown below:

A

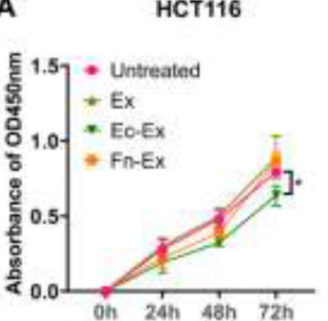

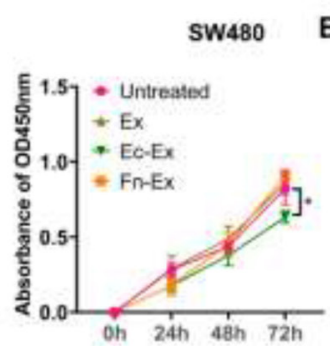

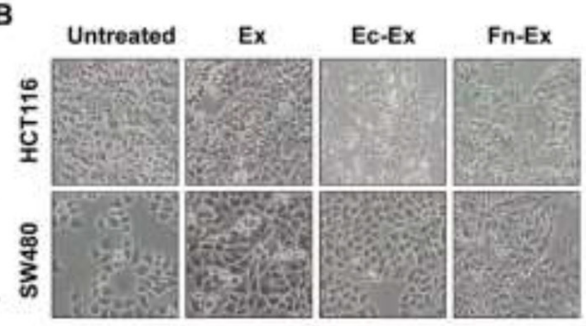

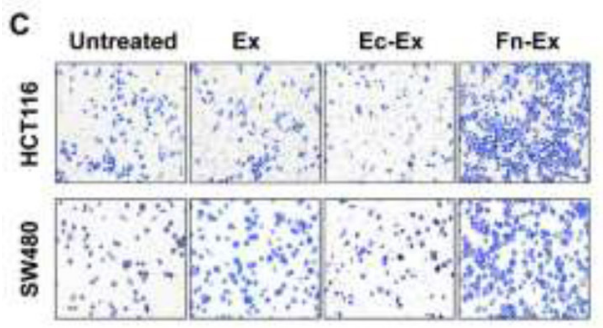
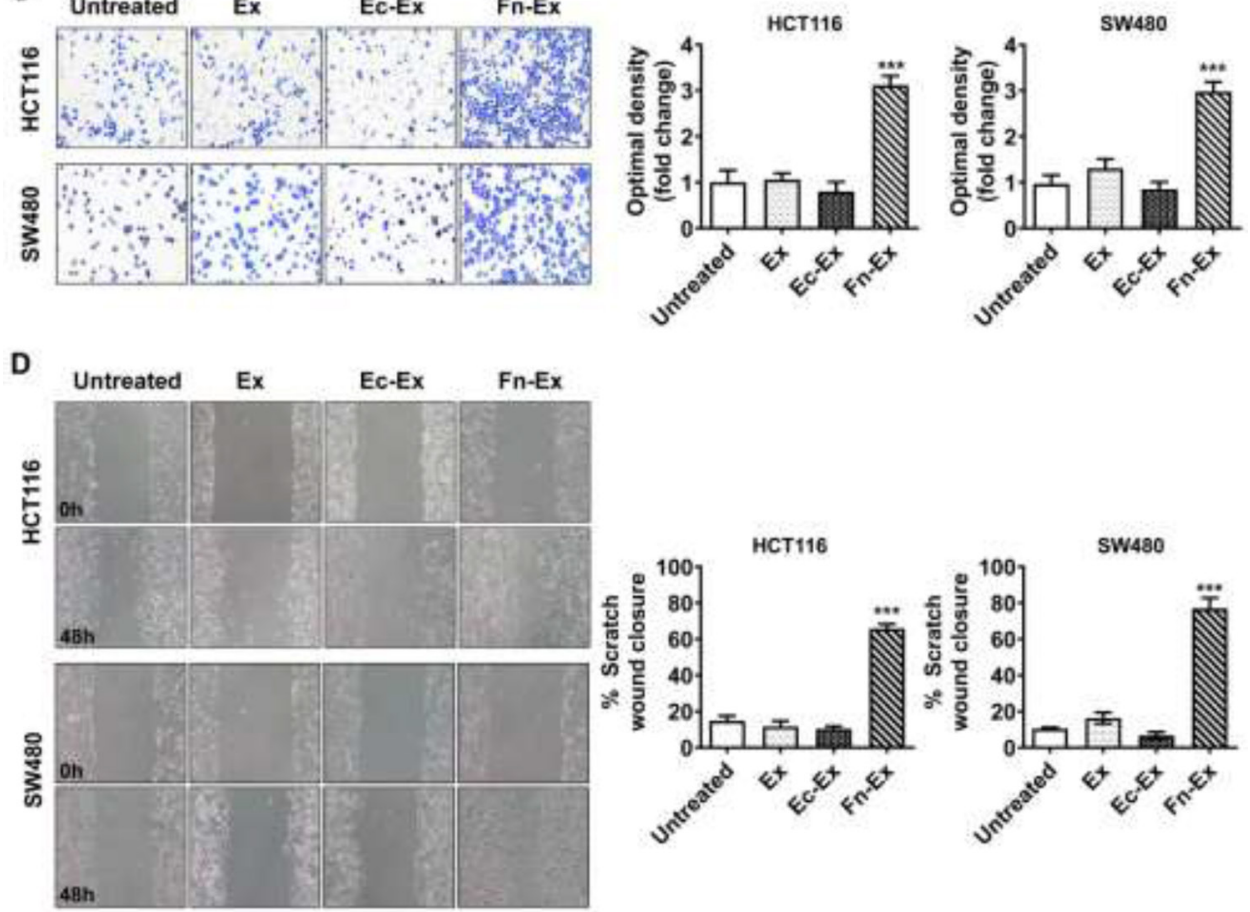

Figure 2 

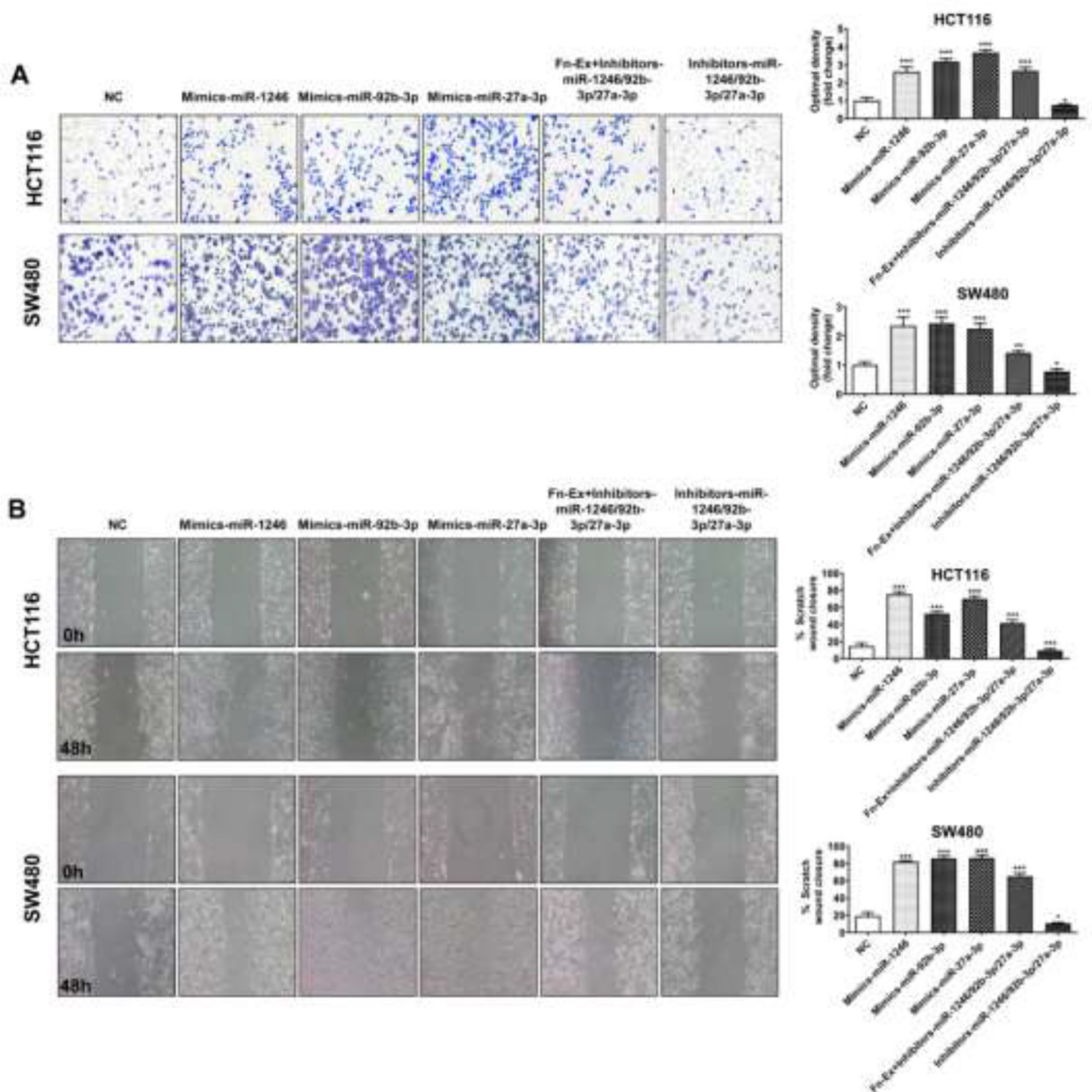

Figure 4 
A

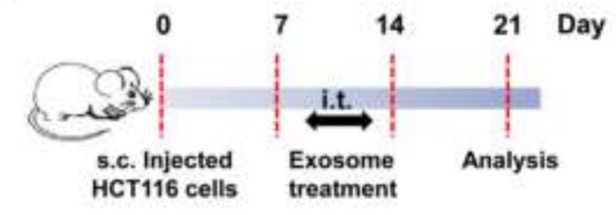

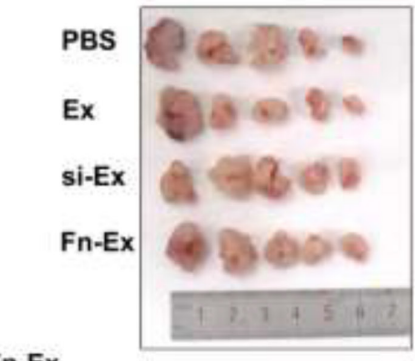

C

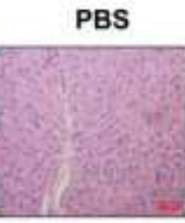

Ex

si-Ex

Fn-Ex

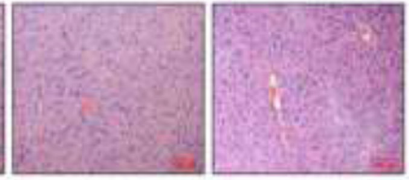

E

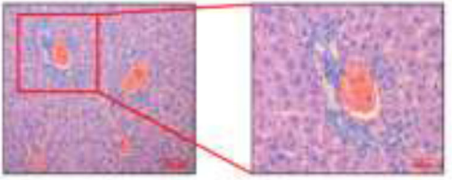

D

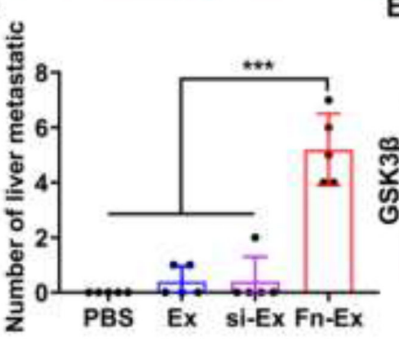

PBS

Ex

si-Ex

Fn-Ex
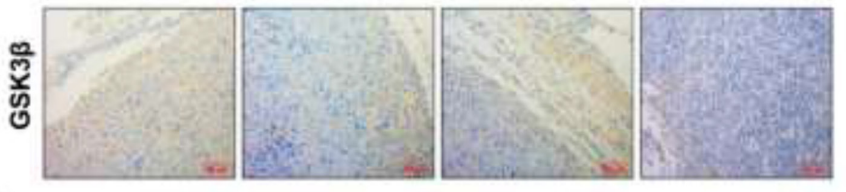

F

PBS Ex Fn-Ex si-Ex

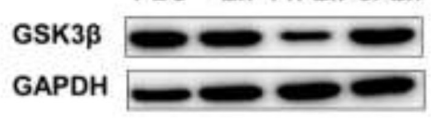

G

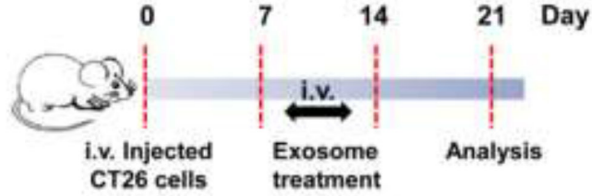

H

J
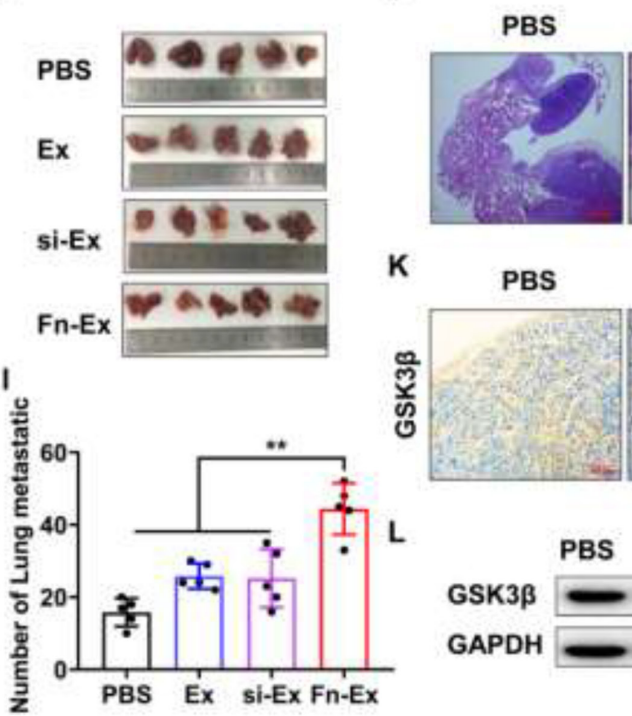

Ex

si-Ex

Fn-Ex
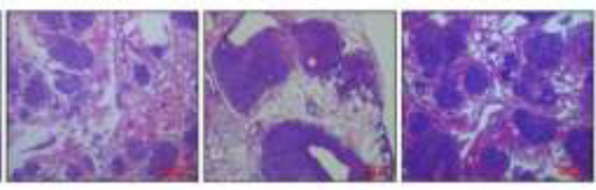

K

PBS

Ex

si-Ex

Fn-Ex

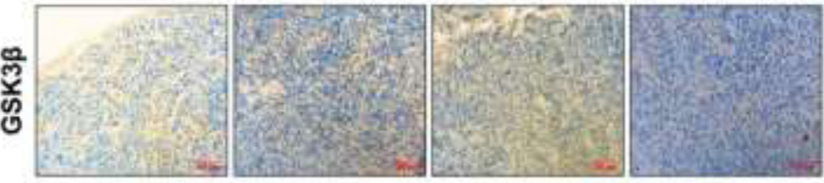

PBS Ex Fn-Ex si-Ex

GSK $3 \beta$

GAPDH

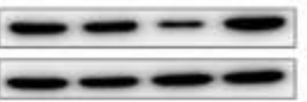

Figure 8

(c) Author(s) (or their employer(s)) 2022. No commercial re-use. See rights and permissions. Published by BMJ. Gut 2022;71:e4. doi:10.1136/gutjnl-2020-321187corr1

(A) Check for updates 